stoff, sondern in anderen Körpern enthalten sind. Da nun die Methode von Rantenberg annähernd das Gewicht des gesammten Stickstoffs des Menschenharns ergibt, so kann sie nicht als quantitative Analyse des Harnstoffs angesehen werden.

Rautenberg's Methode ist also in der That eine Analyse des Stickstoffs. Streng genommen heisst der Titer nicht:

$1 \mathrm{ccm} \mathrm{R}$-Hg-lösung $=0,01 \mathrm{gr}$ Harnstoff, sondern:

$1 \mathrm{ccm} \mathrm{R}^{\prime}$-Hg-lösung $=0,004674 \mathrm{gr}$ Stickstoff.

Wer endlich selbst meine Methode mit der von Rautenberg vergleicht, wird finden, dass der Index bei der letzteren mit weniger Schärfe hervortritt.

(Aus dem physiologischen Laboratorium der Universität Jena.)

\title{
Die Wahrnehmung der Schallrichtung mittelst der Bogengänge.
}

Zum Theil nach Versuchen des Herrn Cand. d. Med. K. Schaefer.

Von

W. Preyer.

Einleitung.

Während es keinem Zweifel unterliegt, dass jede sinnliche Empfindung im Augenblick ihres Auftretens im Bewusstseinsfelde irgendwelchen räumlichen Charakter hat, ist es für sämmtliche Sinnesgebiete und die Organempfindungen fraglich, was die jedem einzelnen Sinneseindruck ausserhalb und innerhalb des Körpers und an seiner Oberfläche zukommende Localisation bestimmt. 
Beobachtungen und einfache Versuche, welche ich vor zehn Jahren zuerst an Säuglingen und sehr jungen Thieren anstellte, um die Entwicklnng des Raumsinnes and die allmähliche Bethätigung des Orientirungsvermögens zu erforschen, lebrten die wichtige Thatsache kennen, dass in einer gewissen fruhen psychischen Entwicklungsphase Thier und Kind ihre eigenen Körpertheile als ihnen selbst fremde Gebilde betrachten ${ }^{1}$ ). Das eintägige Hühnchen pickt nach den eigenen Nägeln wie nach den vorgesetzten Körnern, das kleine Kind beisst sich in den eigenen Arm, sucht einen Finger auszureissen, will einen Fuss von seinem Bein abtrennen. Erst spät werden alle Theile des Körpers als zusammengehörig erkannt und jedem Sinneseindruck ein Ort oder eine Richtung zugewiesen. Es ist dann ganz unmöglich irgend eine bewusste Sinnesempfindung zu haben, welche nicht räumlich mit Bezug auf den eigenen Körper bestimmt wäre, nachdem einmal dieser als Ganzes' von der ïbrigen Welt abgegrenzt worden und den Reiz der Neuheit verloren hat. Aber die Genanigkeit, mit welcher die Localisirung geschieht, ist in den einzelnen Wahrnehmungsgebieten eine sehr ungleiche. Denn während das Auge, vermöge der Innervationsempfindungen seiner Muskeln und seiner grossen Beweglichkeit, sehr kleine Unterschiede der Einfallswinkel erkennt und dadurch ganz nahe, wie Millionen Meilen weit entfernte Körper nebeneinander und übereinander wahrnimmt, ist die Haut nur an wenigen Stellen befähigt, Berührungspunkte von geringem Abstande getrennt zur Perception gelangen zu lassen; die Richtung ungleich temperirter Luftströme wird durch die Haut nur ungenau erkannt, der Ort und Abstand fremder Gegenstände miitelst des Hautsinns ohne Berïhrung nur selten und dann unsicher ermittelt. Die Geschmacksempfindungen werden zwar sämmtlich in die Mundböhle verlegt, in dieser aber nicht weiter genau getrennt, and die Geruchsempfindungen können bezüglich ihrer Herkunft nur höchst unvollkommen local bestimmt werden.

Auch die Organgefühle, wie etwa Hunger- und Durst-Gefühl, Kolikschmerzen, werden sämmtlich nur ungenau localisirt.

Hingegen ist das Gehör im Stande, nicht allein weit entfernte Schallquellen zu erkennen, sondern auch im Inneren des Kopfes Geräusche zu localisiren und sehr kleine Differenzen der

1) Preyer, "Die Seele des Kindes". Lpzg. 1884. 2. Aufl. 
Verschiebungen der Lufttheilchen des äusseren Gehörganges durch Tonhöhenunterschiede zu percipiren. Insofern ist schliesslich die ganze Tonwahrnehmung mittelst des Ohres räumlich, weil es sich dabei um die Distanz zweier erregter Hörnervenenden in der Schnecke handelt, welche mit Leichtigkeit an der Tonhöhendifferenz $\mathrm{zu}$ erkennen ist. Jedoch wird diese beim Menschen hochausgebildete Function zur Raumwabrnehmung und Orientirung nicht verwendet, weil das Gehör in anderer Hinsicht im Stich lässt, nämlich in Betreff der Schallri chtungsperception.

Aus welcher Richtung ein Schallstrahl kommt, wird oft falsch beurtheilt.

Da indessen ïber diese Irrthümer der Gehörsurtheile noch keine genauen und umfassenden Untersuchungen angestellt und im Besonderen die Arten der Verwechslungen garnicht ermittelt worden sind, solche Bestimmungen aber für die Lehre von der Raumwahrnehmung überhaupt die wichtigsten Schlussfolgerungen in Aussicht stellen, so babe ich dem Cand. d. Med. Herrn K. Schaefer aus Rostock vorgeschlagen eine grosse Anzahl von Einzelversuchen zuerst mit mir und dann allein an verschiedenen Individuen, welche gut hören, auszufuhren. Er hat sich mit bemerkenswerthem Gescbick, Eifer und Verständniss dieser Aufgabe gewidmet, so dass genügendes Material an siebzehn gut hörenden und sicher urtheilenden erwachsenen Individuen beiderlei Geschlechts gewonnen wurde, $\mathbf{~ I m}$ die im Folgenden mitzutheilenden Gesetzmässigkeiten und die neue Theorie der Function der Bogengänge zu begründen.

\section{Die Methode.}

Was zunächst die Methode betrifft, so wurde jedes Individuum in derselben Weise geprüft, und zwar, um möglichst alle Einflüsse nebensächlicher Art, Erinnerungen, Ablenkungen der Aufmerksamkeit von der Beurtheilung der Schallrichtung auszuschliessen, mit einem vorher nicht oft gehörten, kurz dauernden, leicht zu erkennenden Schall von möglichst immer derselben Intensität und Qualität in stets fast gleichem Abstande vom Mittelpunkte der die beiden Trommelfelle verbindenden Geraden ${ }^{1}$ ). Ich fand hierzu das früher als Spielzeug benutzte Cri-cri vorzüglich geeignet. Doch

1) Diese Linie hat im Jahre 1800 Venturi die Gehörsaxe oder akustische Axe genannt (Voigt's Magazin für Naturkunde II, S. 3). 
Die Wahrnehmung der Schallrichtung mittelst der Bogengänge. 589

wurde auch das Ticken des Telephons beim Oeffnen und Schliessen eines Stromes von variabler Stärke in einer besonderen Versuchs. reihe verwendet. Beide Schallarten haben den Vortheil, dass sie kurz dauern, also in geringem Abstande vom Kopfe die Reflexion kaum merklich werden kann.

Um möglichst gleiche Abstände der Schallquelle für jede Schallrichtung zu haben, wurde aus Draht eine auf den Kopf zu setzende "Schallhaube" angefertigt, welche starre Drähte von den zu prüfenden Richtungen trägt. Fig. 1, im Einzelnen nicht genau ausgeführt, zeigt wie diese Schallhaube ungefähr aussieht.

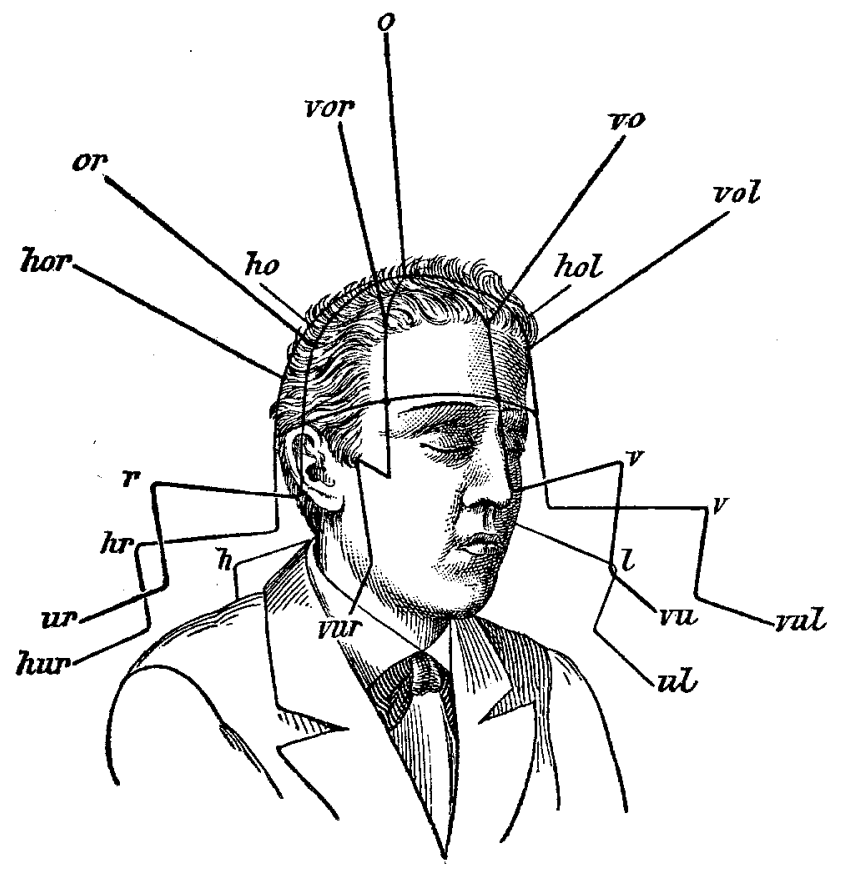

Fig. 1.

Die Zahl der Richtungen beträgt nicht mehr und nicht weniger als 26. Denn man hat:

I) die 6 Endpunkte der 3 primären zu einander senkrechten Axen, nämlich vorn $(\boldsymbol{v})$ und hinten $(\boldsymbol{h})$ für die sagittale Axe, oben $(\boldsymbol{o})$ und unten $(\boldsymbol{u})$ für die verticale und rechts $(\boldsymbol{r})$ und links $(\boldsymbol{l})$ für die transversale (frontale oder Quer-)Axe (Taf. IX, Fig. 1 und 2); 
II) die 12 Endpunkte der 6 secundären Axen, deren je zwei in den, durch die 3 primären Axen bestimmten, zu einander senkrechten Ebenen liegen und sich rechtwinklig schneiden, nämlich:

a) für die sagittale oder Median-Ebene (Taf. IX, Fig. 2) vorn-oben $\boldsymbol{v} o)$ und hinten-unten $(\boldsymbol{h u})$, vorn-unten $(\boldsymbol{v} \boldsymbol{u})$ und hinten-oben (ho),

b) für die transversale oder Frontal-Ebene oben-rechts (or) und unten-links (ul), unten-rechts (ur) und oben-links (ol) (Taf. IX, Fig. 1),

c) für die horizontale Ebene vorn-rechts (vr) und hinten. links $(\boldsymbol{h} \boldsymbol{l})$, vorn-links $(\boldsymbol{v} \boldsymbol{l})$ und hinten-rechts (hr) (Taf. IX, Fig. 3).

III) Die 8 Endpunkte der 4 tertiären Axen, deren jede auf 3 der secundären Axen: vo-hu, vu-ho, or-ul, ur-ol, vr-hl, vl-hr senkrecht steht, nämlich (Taf IX, Fig. 4 and 5):

a) vorn-oben-rechts (vor), hinten-unten-links (hul)

b) vorn-oben-links (vol), binten-unten-rechts $(\boldsymbol{h} \boldsymbol{u} \boldsymbol{r})$

c) hinten-oben-rechts (hor), vorn-unten-links (vul)

d) hinten oben-links (hol), vorn-unten-rechts (vur).

Diese 26 Schallrichtungen oder 13 Axen sind anf Tafel IX in 5 Figuren mittelst 5 Ebenen anschaulich dargestellt.

\section{Veranschaulichungsmittel.}

Da bei der Beurtheilung der Richtung, aus welcher ein Schall auf den aufrecht stehenden oder sitzenden Menschen zukommt, weniger als diese 26 Ausdrücke nicht ausreichen und mehr nicht oft gebraucht werden, so erschien es zweckmässig weitere Unterabtheilungen, wie etwa beim Compass, einstweilen nicht zu unterscheiden. Es wäre jedoch leicht, geringere Differenzen der Schallrichtungen mittelst der vorstehenden Terminologie ebenso bestimmt zu bezeichnen, wie in der Sprache der Seeleute die Windrichtnngen bezeichnet werden. Zum Beispiel würde der Richtung „WNW nach W" entsprechen ein Schall lol nach $l$, d. h. links-obenlinks nach links, und so in allen Ebenen.

Uebrigens ist es nicht leicht die 13 aufgezählten Axen anschaulich in der Vorstellung zu haben; daher empfiehlt es sich, das ganze System an bestimmte Körper geknüpft im Gedächtniss zu behalten. Der reguläre enteckte entkantete Würfel und das reguläre enteckte und entkantete Oktaëder sind solche Sechsund- 
zwanzigflächner. Die Mittelpunkte der 26 Flächen bilden die 26 Endpunkte der genannten 13 Axen (Fig. 2 und Fig. 3):

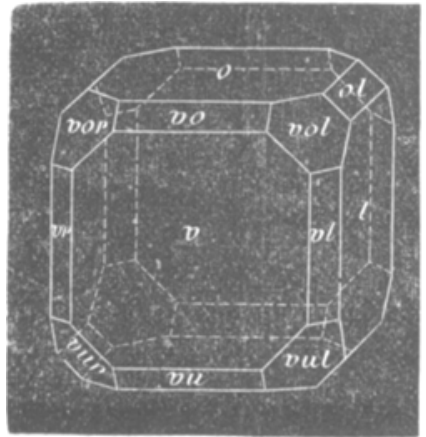

Fig. 2.

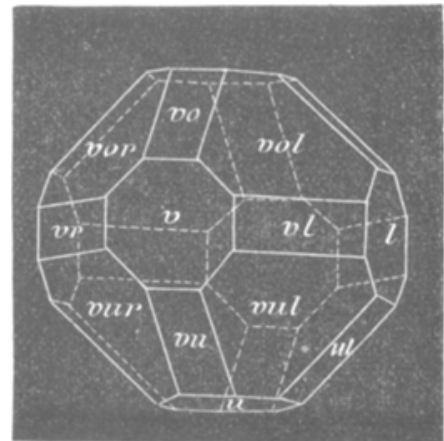

Fig. 3.

Um die Neigung der 13 Axen zueinander und ibre Ordnung jeden Augenblick gegenwärtig zu haben, ist es rathsam ein kleines Modell (Fig. 4) anzufertigen, in welchem die primären, secundären und tertiären Axen von ungleicher Dicke und Farbe, jedoch von gleicher Länge sind - letzteres um leichter grösste Kreise mit einem Ring veransehaulichen zu können.

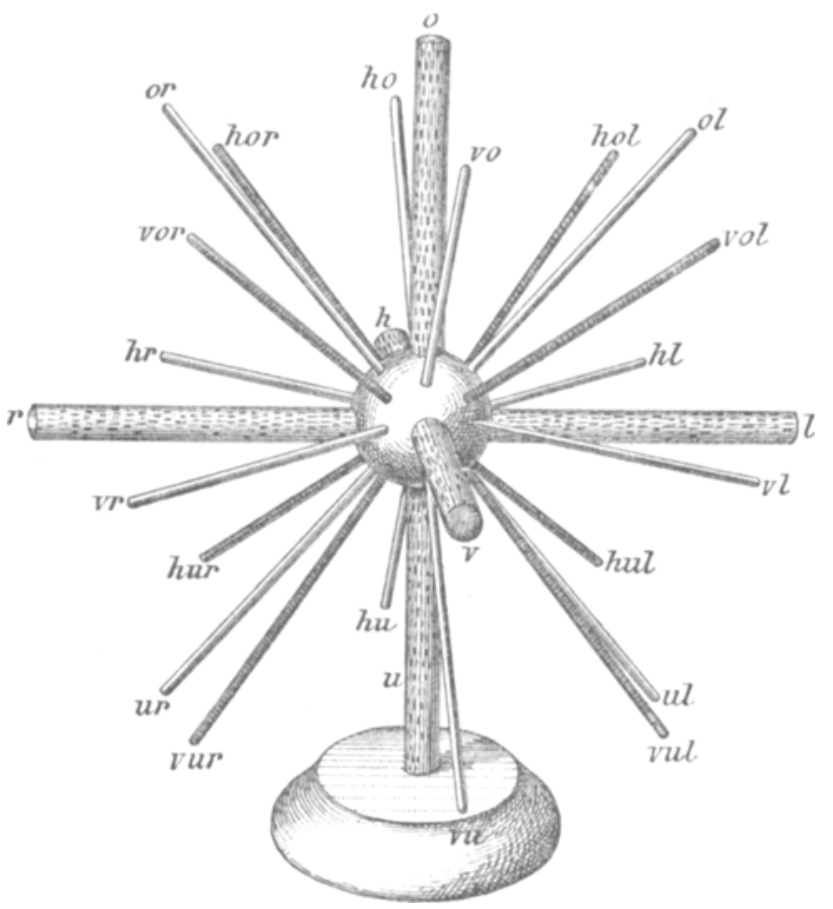

Fig. 4. 
Bei den vorliegenden Versuchen kommt es nun vor Allem auf die Anzahl der richtigen Fälle, die Art, die Anzahl und die Grösse der Fehler an. Jene ergeben sich aus den Protokollen unmittelbar, da in den für diese Versuche angefertigten lithographirten Formularen neben die wahren, ein für allemal vorgedruckten Schallrichtungen die bei fest verschlossenen Augen in unbewegter sitzender Haltung wahrgenommenen geschrieben werden; die Grösse der Fehler wird gemessen durch die Winkel, welche die wahre Schallrichtung mit der vermeintlichen bildet. Es mussten also alle Winkel, welche die sich im Axencentrum durchschneidenden 26 Schallrichtungen miteinander bilden, vorerst ermittelt werden.

Trägt man auf die Oberfläche eines nicht zu kleinen, genau kugelförmigen Globus die 26 Punkte auf und verbindet man je 2, also mit den antipodischen Gegenpunkten je 4 oder 2 Paare, durch grösste Kreise miteinander, so gewährt die Oberfläche der Kugel ein anschanliches Bild der Fehlergrössen, welche in Graden auf die Seiten der durch die grössten Kreise gebildeten sphärisehen Dreiecke eingeschrieben werden können (Fig. 5). Dieser „Schall-

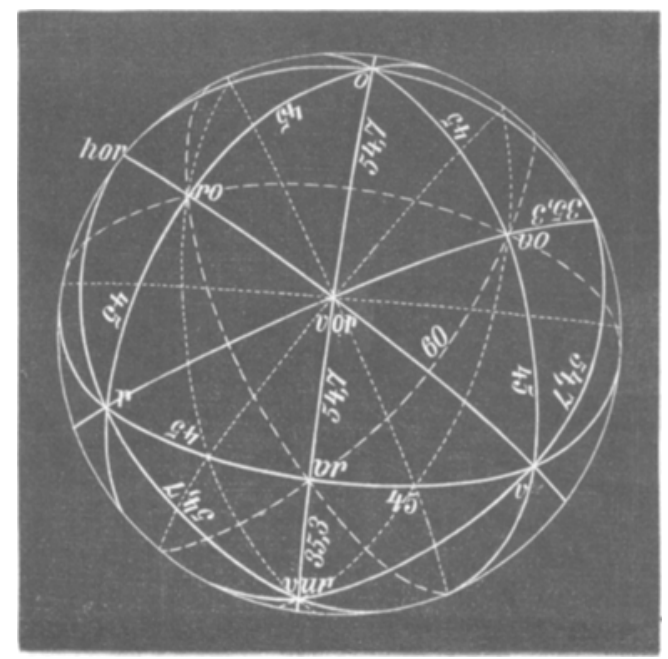

Fig. 5.

globus" gestattet schneller als die folgende, alle vorkommenden Fehler nmfassende Tabelle die Anzahl von Graden abzulesen, um welche man irrte. (Siehe Tabelle I.) 


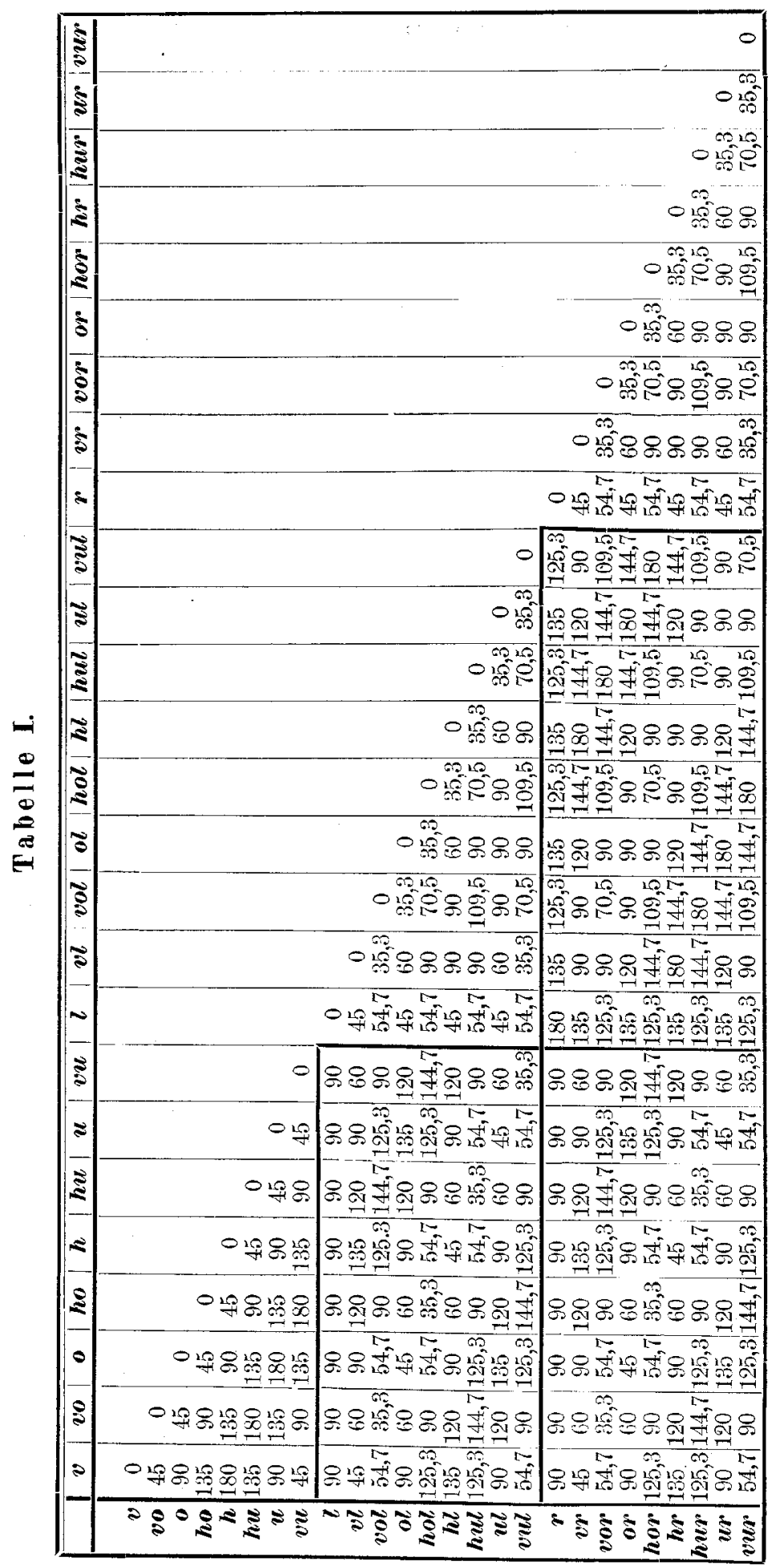




\section{Die möglichen Verwechslungen.}

Es bedarf keiner Erläuterung, dass im Ganzen $26^{2}-26=650$ Verwechslungen the oretisch möglich sind. Die Hinzufitgung der 26 riehtigen Fälle (mit dem Fehler von $0^{0}$ ) liefert die Gesammtzahl von 676 Einzelfällen, welche bestehen aus

325 specifisch verschiedenen Fehlern

325 damit identischen ungleichsinnigen

26 richtigen Fällen

\section{6}

Wenn z. B. vorn-unten für hinten-oben erklärt wird, so folgt daraus nicht, dass auch der wahre Schallort hinten oben für vornunten erklärt wird. Der Fehler wäre in beiden Fällen identisch, aber ungleichsinnig. Aus der Thatsache, dass einige Fehler nur in dem einen Sinne gemacht werden, folgt die Nothwendigkeit, behufs Registrirung aller Febler, die leeren Felder der Tabelle entsprechend den ausgefüllten - mit den gleichen Winkelgrössen auszufüllen, was hier nur der Uebersichtlichkeit wegen unterblieb.

Im Ganzen hat man nur zwölf Fehlergrössen; nämlich einen Fehler von

\begin{tabular}{|c|c|c|c|}
\hline 180 & Grad & 26 & mal \\
\hline 144,7 & $"$ & 48 & $"$ \\
\hline 135 & $"$ & 48 & " \\
\hline 125,3 & 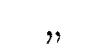 & 48 & $"$ \\
\hline 120 & 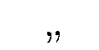 & 48 & $"$ \\
\hline 109,5 & $\eta$ & 24 & $n$ \\
\hline 90 & $n$ & 192 & , \\
\hline 70,5 & 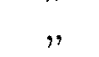 & 24 & $"$ \\
\hline 60 & $n$ & 48 & $n$ \\
\hline 54,7 & 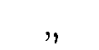 & 48 & $n$ \\
\hline 45 & $\because$ & 48 & $"$ \\
\hline \multirow[t]{2}{*}{35,3} & ", & 48 & $"$ \\
\hline & & \multicolumn{2}{|c|}{$650=2.325$} \\
\hline $\begin{array}{ll}n & 0\end{array}$ & Grad & 26 & mal \\
\hline
\end{tabular}

Wenn nun mittelst der beiderseits gleich scharfen Gehörorgane allein, obne alle Gesichtseindrücke, Kopfbewegungen, Berührungen, Suggestionen a. a. m. gar keine Wahrnehmung der 
Schallrichtung möglich wäre, dann müssten in einer sehr grossen Vèrsuchsreihe alle jene 676 Fälle vorkommen, im Besonderen nur etwa $4 \%$, genauer von 26 Fällen nur einer, also 3,85\%, richtig sein, jedenfalls wären unter 1000 Rathversuchen durchschnittlich keine' 40 genau richtig.

\section{Die richtigen Urtheile.}

Ich habe das erste und zweite Tausend unserer genau protokollirten, völlig zuverlässigen, an Normalhörigen, mit geschlossenen Augen in unbewegter, sitzender Haltung gewonnenen Bestimmungen gepruft und gefunden, dass bei gleicher Anzahl von Fällen für jede der 26 Schallrichtungen mit gleicher Schall-Distanz und Stärke - nur "unten" weicht hierin ab wegen der Nothwendigkeit den Schall unter dem Sitz hervorzurufen - nicht $4 \%$, sondern $29,4 \%$ aller Versuche ein genau richtiges Resultat liefern; Der Zufall ist somit ausgeschlossen. Mittelst der Ohren allein kann vielmehr ohne irgendwelche Beihülfe anderer Sinnesorgane and ohne Kopfbewegungen in mehr als einem Viertel aller Fälle die Schallrichtung genau angegeben werden. Von 2080 gleichwerthigen Urtheilen sind 612 richtig, und zwar ist dabei jede der 26 Richtungen 80 mal beurtheilt worden.

Die richtigen Fälle vertheilen sich jedoch in sehr ungleicher Weise auf die 26 versehiedenen Schallrichtungen.

Es wurden genau richtig beurtheilt unter je 80 Fällen:

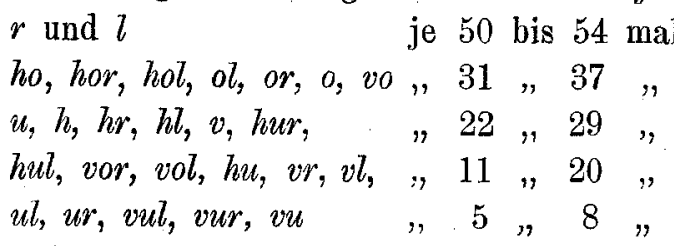

Falsche Urtheile kamen vor unter je 80 Fällen:

26 bis 30 bei $r$ und $l$

43 " 49 , ho, hor, hol, or, ol, o, vo

$51,58, u, " h, h r, h l, v, h u r$

60,69, hul, vor, vol hu, vr, vl,

72,75, , ul, ur, vul, vur, vu

and zwar ist unter mehrerentausend Versuchen an normal hörenden Menschen nicht. ein einzigesmal ein von links kommender Schall nach rechts verlegt worden und umgekehrt. 
Hieraus folgt mit Sicherheit, dass rechts und links durch das Gehör allein viel leichter richtig wahrgenommen werden, als oben und unten und hinten und vorn.

Es ergeben sich für die transversale Axe $(r l)$ und die verticale (ou) und die sagittale Axe ( $h v$ ) folgende Zahlen an völlig richtigen Urtheilen unter je 160 Proben:<smiles></smiles>

104 Fälle $=65,0 \% \quad 62$ Fälle $=38,8 \% \quad 50$ Fälle $=31,2 \%$ dagegen an völlig richtigen Urtheilen für die je 8 Schallrichtungen in den drei primären Ebenen:

in der transversalen Ebene (Fig. I) $38,7 \%$ d. i. 248 Fälle, in der horizontalen Ebene (Fig. III) $35,8 \%$ d. i. 229 Fälle, in der sagittalen Ebene (Fig. II) $31,5 \%$ d. i. 202 Fälle.

In der sagittalen Ebene fällt der Vortheil, den die Beurtheilung des rechts und links in den beiden anderen Ebenen mit sich bringt, fort, daher der Abfall '). So stark ist der Einfluss des $r$ und $l$, dass sogar die Summe aller richtigen Fälle derjenigen 18 Schallrichtungen, an denen $r$ und $l$ betheiligt sind, procentisch eine grössere, als die der 18 ou- und der $18 \mathrm{hv}$-Richtungen liefert, da eben in letzteren beiden $r$ und $l$ nicht so oft vertreten sind. Man hat richtige Urtheile:

mit links und rechts $28,4 \%$ d. i. 410 von 1440

mit oben und unten 26,6, d. i. 386 von 1440 .

mit vorn und hinten 25,6 , d. i. 369 von 1440

Ferner ist die Beurtheilung der Schallrichtungen, welche irgendwoher von oben kommen, bei weitem sicherer, als die der von unten kommenden. Denn die Zahlen der richtigen Fälle sind für ho, hor, hol, or, ol, o, vo, vor, vol, also für oben $37,6 \%$ oder 271 , für unten 15,5\% oder 112 Fälle; letztere umfassen $u$, hur, hul, $h u, u l, u r, v u l, v u r, v u$.

Endlich wird eine Schallrichtung, an der $h$ betheiligt ist, viel besser erkannt, als eine solche an der $v$ theilnimmt. Die Anzahl der richtigen Fälle ist hier für $h o$, hor, hol, $h, h r, h l$, hur, $h u l$, hu, also für hinten 234 , d. i. $32,5 \%$, für vorn, d. h. für vo, vor, vol, $v, v r, v l, v u r, v u l, v u$ 130, d. i. $18,0 \%$.

1) Dass die transversale Ebene vor der horizontalen etwas bevorzugt ist, findet seinen Grund in der Bevorzugung der von $o$ und $u$ kommenden Schalleindrücke gegenüber denen, die von $h$ und $v$ kommen. 
Diese Versuchsergebnisse werden bestätigt und ergänzt durch die nähere Untersuchung der Fehler.

\section{Die Arten der Verwechslung.}

Wenn man erwägt, dass 25 Verwechslungen einer jeden der 26 Schallrichtungen mit einer anderen von vornberein theoretisch möglich erschienen, so nimmt es Wunder, dass bei keiner mehr als 8 verschiedene Verwechslungen vorkommen. Von den denkbaren 299 (d. i. 325-26) Verwechslungsarten sind überhaupt innerhalb der ersten zweitausend Bestimmungen nur 96 vorgekommen und davon mehrere nur je einmal. Ich kann sogar mit Bestimmtheit vorhersagen, dass durch Vervielfältigung der Versuche an normal Hörenden nicht mehr als 100 Fehlerarten von den 299 als thatsächlich vorkommend erwiesen werden können, oder, was dasselbe ist, wenn man die ungleichsinnigen Fehler doppelt nimmt, werden von den denkbaren 650 Fehlerarten nicht mehr als höchstens 200 beobachtet werden. Ich setze dabei beiderseits gleiche Hörschärfe, normales Urtheilsvermögen und genaues Experimentiren an bilateral-symmetrischen Köpfen voraus.

Hält man sich nämlich an das bis jetzt verarbeitete Material, so ergeben sich folgende Thatsachen.

1) Jede der 26 geprüften Schallrichtungen wird richtig erkannt - freilich einige, wie $v u$, nur sehr selten, einzelne, wie $l$ und $r$, in $2 / 3$, andere in $1 / 3$ aller Fälle bei gleichen Versuchsbedingungen. Hierdurch fallen 26 richtige Fälle von den 676 fort.

2) Kein normaler Beobachter verlegt, wie bereits hervorgehoben wurde, einen die linke Kopfhälfte treffenden Schalleindruck in die rechte und keinen die rechte treffenden in die linke. Da $9 l$-Richtungen und $9 r$-Richtungen vorliegen, so fallen 2 mal 81 Verwechslungsarten, d. h. 162 Fehlerarten fort. Denn kein Glied der Reihe $l, r l, v o l, o l$, hol, $h l, h u l, u l, v u l$ wird mit irgend einem der Reihe $r$, vr, vor, or, hor, hr, hur, ur, vur verwechselt.

3) Kein aufmerksamer normaler Beobachter verlegt einen die

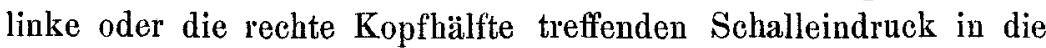
Medianebene, $d$. b. kein Glied der beiden eben genannten Reihen wird für ein Glied der sagittalen Reihe

$$
v, v o, o, h o, h, h u, u, v u
$$

gehalten. Hierdurch fallen $2 \mathrm{mal} 8 \mathrm{mal} 9$ Verwechslungen fort, d. h. 144 Fehlerarten. Dieser Satz ist jedoch nicht so zu ver- 
stehen, als wenn bei der gewählten Art der Hörprüfung überhaupt kein von $l$ oder $r$ kommender Schall in die Medianlinie verlegt würde, sondern er gilt nur für exactes Experimentiren und gute Beurtheiler. Anfangs, ehe ich bemerkt hatte, dass diese auf Nachlässigkeit im Urtheilen beruhenden Verwechslungen bei manchen uberhaupt nicht, oder nur ausserordentlich selten, vorkommen, wurden sie den anderen Verwechslungen gleichgeachtet, mussten aber später cassirt werden, da sie jedesmal revocirt wurden, wenn nur der Schallort genau innegehalten und ein aufmerksames Urtheil verlangt wurde. Derartige Verwechslungen sind denn auch in den letzten Centurien gar nicht mehr vorgekommen.

Dasselbe gilt in jeder Hinsicht von dem folgenden Satze:

4) Kein aufmerksamer, normaler Beobachter verlegt einen, einen beliebigen Punkt der Medianperipherie des Kopfes treffenden Schalleindruck nach links oder nach rechts, d. h. kein Glied der Reihe

$$
v, v o, o, h o, h, h u, u, v u
$$

wird für irgend ein Glied der beiden Reiben

$$
\begin{aligned}
& \text { vl, vol, ol, hol, hl, hul, ul, vul } \\
& \text { vr, vor, or, hor, hr, hur, ur, vur }
\end{aligned}
$$

gehalten.

Hierdurch fallen abermals 2 mal 8 mal 9 Verwechslungen fort, d. b. 144 Fehlerarten.

Handelt es sich also um Ermittlung der wirklich vorkommenden Verwechslungen, so müssen von den 676 Fällen sogleich in Abzug gebracht werden

26 richtige Urtheile

162 Verwechslungen von $l$ und $r$

144 Verlegungen von $l$ und $r$ in die Medianebene

144 Verlegungen aus der Medianebene nach $l$ und $r$.

476

Somit bleiben nur $676-476$, d. h. 200 Fehlerarten oder 100 Verwechslungen im Ganzen, wenn man das Urtheil „den Schall $a$ höre ich bei $b^{\text {" }}$ mit dem anderen Urtheil „den Schall $b$ höre ich

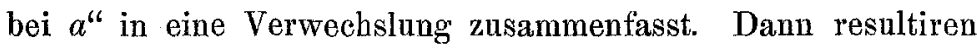

351 Fälle $(325+26)$, 
davon 26 richtig

81 gekreuzt $l r$

72 von $r$ und $l$ in die Medianebene

72 von der Medianebene nach $r$ und $l$

251 nicht wirkliche Verwechslungen.

Somit $351-251=100$ Fehlerarten.

Von diesen kommen auf die linke und die rechte Kopfhälfte mit je 9 Richtungen je 36 Verwechslungen, welche sämmtlich beobachtet sind, und auf die Peripherie der Sagittalebene des Kopfes mit 8 Richtungen 28, von denen 24 in den Protokollen verzeichnet stehen. Die 4 feblenden Verwechslungsarten sind:

$$
\begin{aligned}
& v \text { und } u \\
& o \text { und } u \\
& v \text { und } h u \\
& v o \text { und } u \text {. }
\end{aligned}
$$

Es ist wahrscheinlich, dass diese Verwechslungen iberhaupt sehr selten vorkommen, bei unseren Versuchen aber zum Theil deshalb fehlten, weil die Richtung $u$ im Vergleich zu allen anderen Richtungen etwas bevorzugt war. Der Schall musste stets unter dem Sitz des Urtheilenden erzeugt werden (s. o. S. 605), was der letztere an der geringeren Intensität erkennt.

Es ist aber, wie sich weiter unten zeigen wird, ganz abgesehen von dieser Besonderheit unwahrscheinlich, dass oft $v$ fïr $u$ und $u$ für $v$, dass $o$ für $u$ und $u$ für $o$, dass $v$ für $h u$ und $h u$ für $v$, und dass $v o$ für $u$ und $u$ für $v o$ gehalten wird, so oft auch $v u$ für $v o$ und hu für ho erklärt werden kann.

Jedenfalls bleiben für die Zukunft, wenn man das hier gewählte Verfahren beibehält, nur 96 bis höchstens 100 Fehlerarten bestehen, nämlich die oben genannten 24 (28) medianen, 36 linksseitigen und 36 rechtsseitigen Verwechslungsarten.

Ueber die Vertheilung dieser thatsächlich festgestellten Fehlerarten gibt die folgende Uebersicht Aufschluss:

24 mediane Verwechslungen

$$
\begin{aligned}
& v \text { und } v o, o, v u, h o, h \\
& v o ", \quad o, v u, h o, h u, h \\
& o \quad, \quad h o, h, v u, h u \\
& h o, \quad h, h u, v u, u \\
& h \quad " \quad h u, u, v u \\
& h u \Rightarrow \quad u, v u \\
& u \quad " v u
\end{aligned}
$$


36 Verwechslungen der linken Kop hbälfte

$l$ und $u l, h l, v l, o l, v u l, h o l, v o l, h u l$

vl , ol, vol, vul, hl, hol, $u l, h u l$

vol ," hol, ol, vul, hl, hul, ul

ol ", hol, $h l, v u l, u l, h u l$

hol " hl, hul, vul, ul

$h l, " h u l, u l, v u l$

$h u l$, , $u l, v u l$

$u l, v u l$

36 Verwechslungen der rechten Kopfhälfte

$r$ und ur, vr, hr, vor, or, vur, hor, hur

vr ", vor, vur, or, hr, hor, ur, hur

vor ", or, hr, vur, hor, hur, ur

or " hor, hr, vur, ur, hur

hor ", hr, hur, vur, ur

hr , hur, ur, vur

hur , ur, vur

ur , vur

In dieser Zusammenstellung ist keine Rücksicht genommen darauf, ob die einzelnen Verwechslungen nur in einem Sinne oder auch im entgegengesetzten vorkommen. Sie soll nur angeben, dass die betreffenden Schallrichtungen miteinander verwechselt werden. Zum Beispiel ist der wahre Schallort $v l$ einmal für hul gehalten worden, aber der wahre Schallort $h u l$ ist keinmal für $v l$ erklärt worden. Dagegen wurde ho $12 \mathrm{mal}$ für $o$, und $o 23$ mal für ho gehalten. Beidesfalls ist hier je eine Verwechslungsweise statuirt worden, um zunächst die Natur der überhaupt, so oder so, vorkommenden Verwechslungen zu fixiren.

Innerhalb jeder Zeile der Zusammenstellung ist ferner insofern die Häufigkeit der Verwechslungsarten angedeutet, als die am hänfigsten beobachteten links stehen und die weniger häufigen in der Mitte abnehmend nach rechts, so dass die äussersten Glieder rechts auf jeder Zeile die seltensten Verwechslungen angeben.

Es ergibt sich schon aus dieser Anordnung ein merkwürdiger Parallelismus der 3 Gruppen, sofern mit einigen unerheblichen Platzvertausehungen benachbarter Glieder die erste Gruppe ohne Aenderung der Orte ihrer Glieder in jeder der beiden anderen sich wiederfindet, wenn man jedem ihrer Glieder ein $l$, beziehentlich ein $r$ anhängt, das heisst: die Verwechslungen, welche in 
Die Wahrnehmung der Schallrichtung mittelst der Bogengänge. 601

\begin{tabular}{|c|c|c|}
\hline 5 & $\cdot \cdot \cdot \cdot \cdot \cdot \cdot \cdot \cdot \cdot \cdot \cdot \cdot \cdot \cdot 10$ & \\
\hline 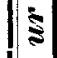 & ........................... & 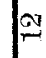 \\
\hline 15 & ....................... & $1 \%$ \\
\hline$\vdots$ & $\cdots \cdots \cdots \cdots \cdots \cdots \cdots$ & 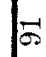 \\
\hline ఏ్s & 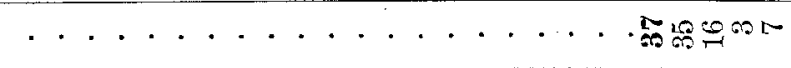 & $\$$ \\
\hline$\delta^{2}$ & $\cdots \cdots \cdots \cdots \cdots \cdots$ & 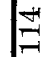 \\
\hline ș & 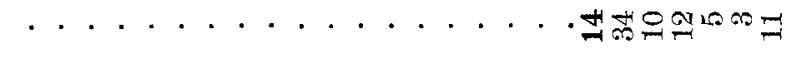 & $\infty$ \\
\hline 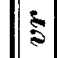 & 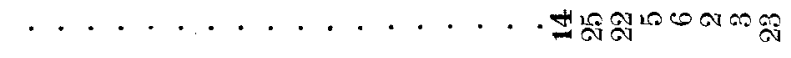 & 8 \\
\hline$\&$ & 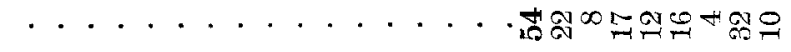 & 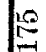 \\
\hline ई & 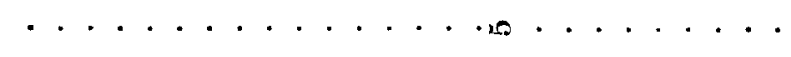 & 10 \\
\hline$\widehat{s}$ & 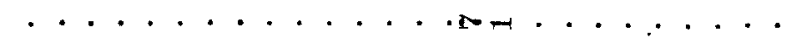 & $\infty$ \\
\hline گa & 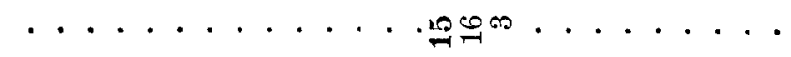 & in \\
\hline 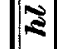 & 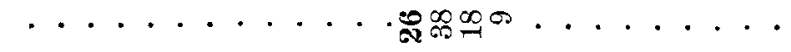 & Fs \\
\hline$\vec{\xi}$ & 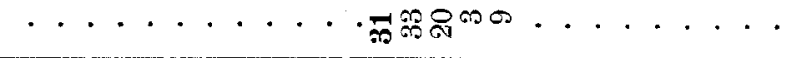 & $\%$ \\
\hline $\overrightarrow{0}$ & $\cdot \cdot \cdot \cdot \cdot \cdot \cdot \cdot \cdot \cos 8$ & 佥 \\
\hline 2 & 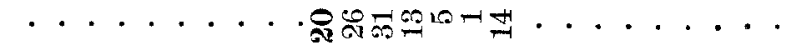 & 胥 \\
\hline$\vec{z}$ & 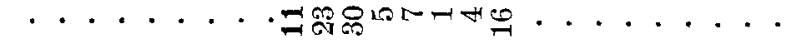 & 5 \\
\hline$\sim$ & ................ & 焉 \\
\hline ริ & 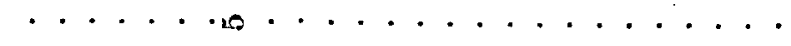 & مـ \\
\hline$\approx$ & 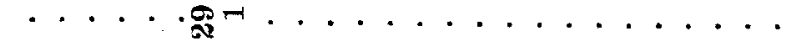 & 命 \\
\hline 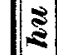 & 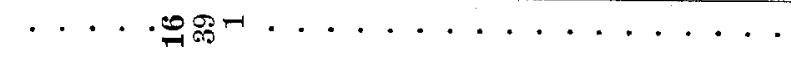 & 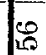 \\
\hline 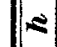 & 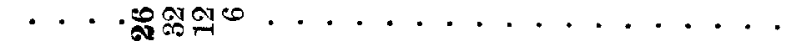 & 0 \\
\hline$\$$ & 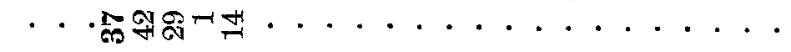 & הి \\
\hline 10 & 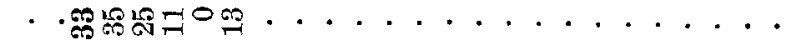 & 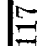 \\
\hline is & 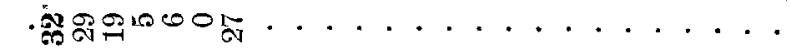 & $\underset{1}{-\infty}$ \\
\hline 2 & 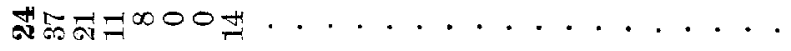 & 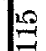 \\
\hline & 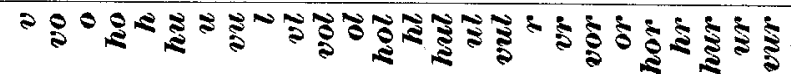 & 8 \\
\hline
\end{tabular}


der Medianebene stattfinden, werden in annähernd derselben Häufigkeit in der linken und in der rechten Kopfhälfte beobachtet, falls unter sonst gleichen Versuchsbedingungen die Schalleindrücke nur die eine Kopfhälfte oder die eine stärker als die andere treffen.

Die Tabelle II enthält die 'Zahlen, welche die Anzahl der wirklich vorkommenden Verwechslungen angeben. Jede Schallrichtung wurde 80 mal geprüft. Nach der Häufigkeit der Verwechslungen dieser 2080 Einzelversuche orduen sich dieselben in folgende Reihe. Es wurden verwechselt

Tabolle III.

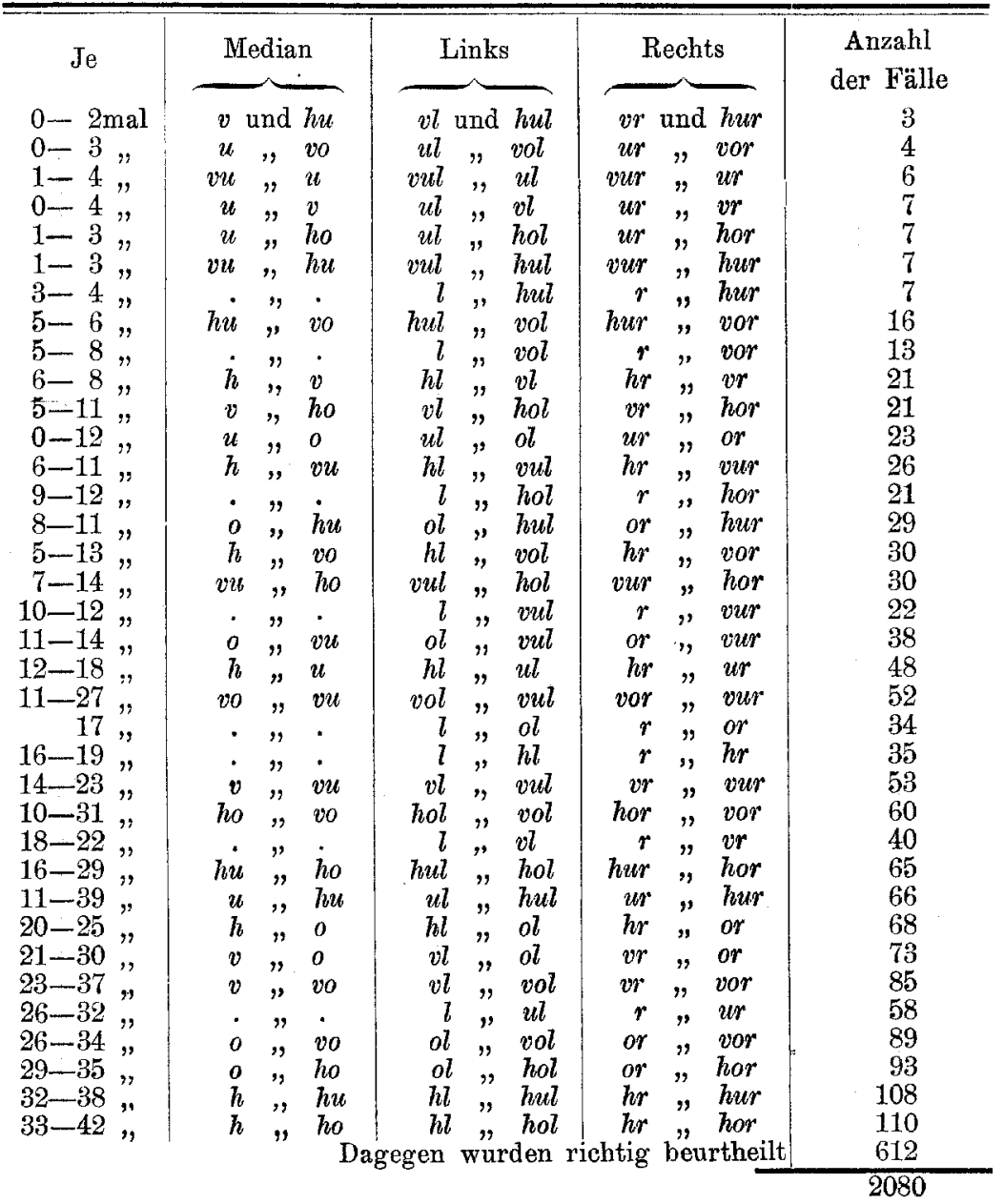


Die Wahrnehmung der Schallrichtung mittelst der Bogengänge. 603

Hierdurch wird der obige Satz vollständig thatsächlich begründet. Die Verwechslungen der nur die linke oder nur die rechte Kopfhälfte treffenden Schallrichtungen lassen sich, sofern es sich nicht um Verwechslungen handelt, bei welchen rein $r$ und $l$ betheiligt sind, aus denen der Medianebene bezüglich der Häufigkeit ihres Vorkommens vorausbestimmen, wenn der zu Prïfende beiderseits gleiche Hörschärfe hat und aufmerksam urtheilt.

Ferner lehrt die Tafel II (S. 611) und die übersichtliche Zusammenstellung (S. 612), dass einige Schallriehtungen nur sehr selten oder garnicht miteinander verwechselt werden, z. B. wurden $u$ und $v, u$ und $o, u$ und $v o, v$ und $h u$ keinmal und die entsprechenden Richtnngen mit $l$ und $r$ nur selten miteinander verwechselt. 'Dagegen wurden viele Schallrichtungen öfter mit anderen verwechselt, als richtig erkannt; so wird gehalten

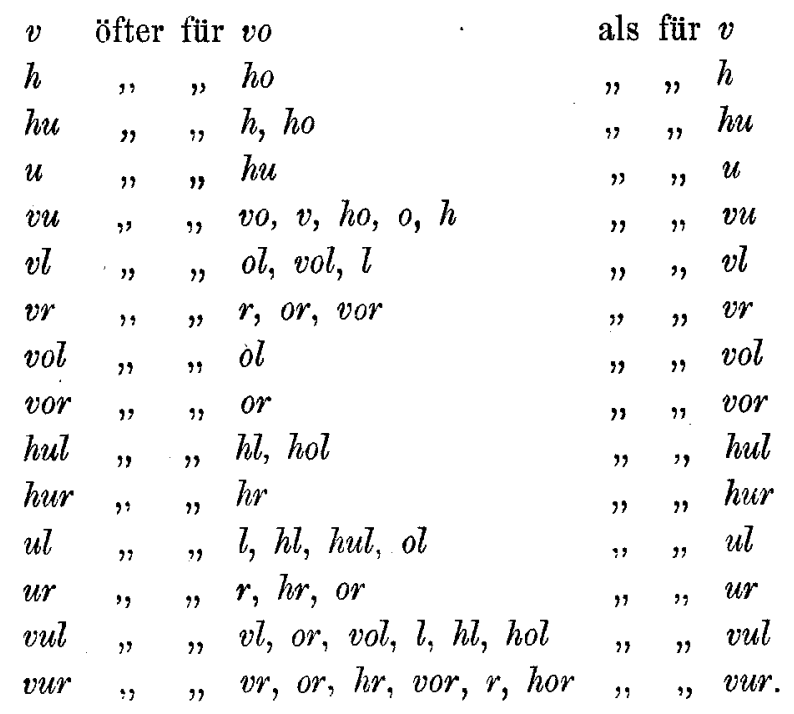

Die folgende Tabelle enthält die unmittelbaren Versuchsergebnisse, welche diese Unterschiede beweisen. Die wahren Schallorte stehen links tibereinander; die Anzahl der richtigen und falschen Urtheile findet man in der horizontalen Richtung unter den uiber den Zahlen verzeichneten Schallorten. Also liegen hier nur einsinnige Verwechslungen vor. Die Richtungen links werden gehalten für die rechts, nicht umgekehrt. Jede Richtung warde 80 Mal geprüft. 


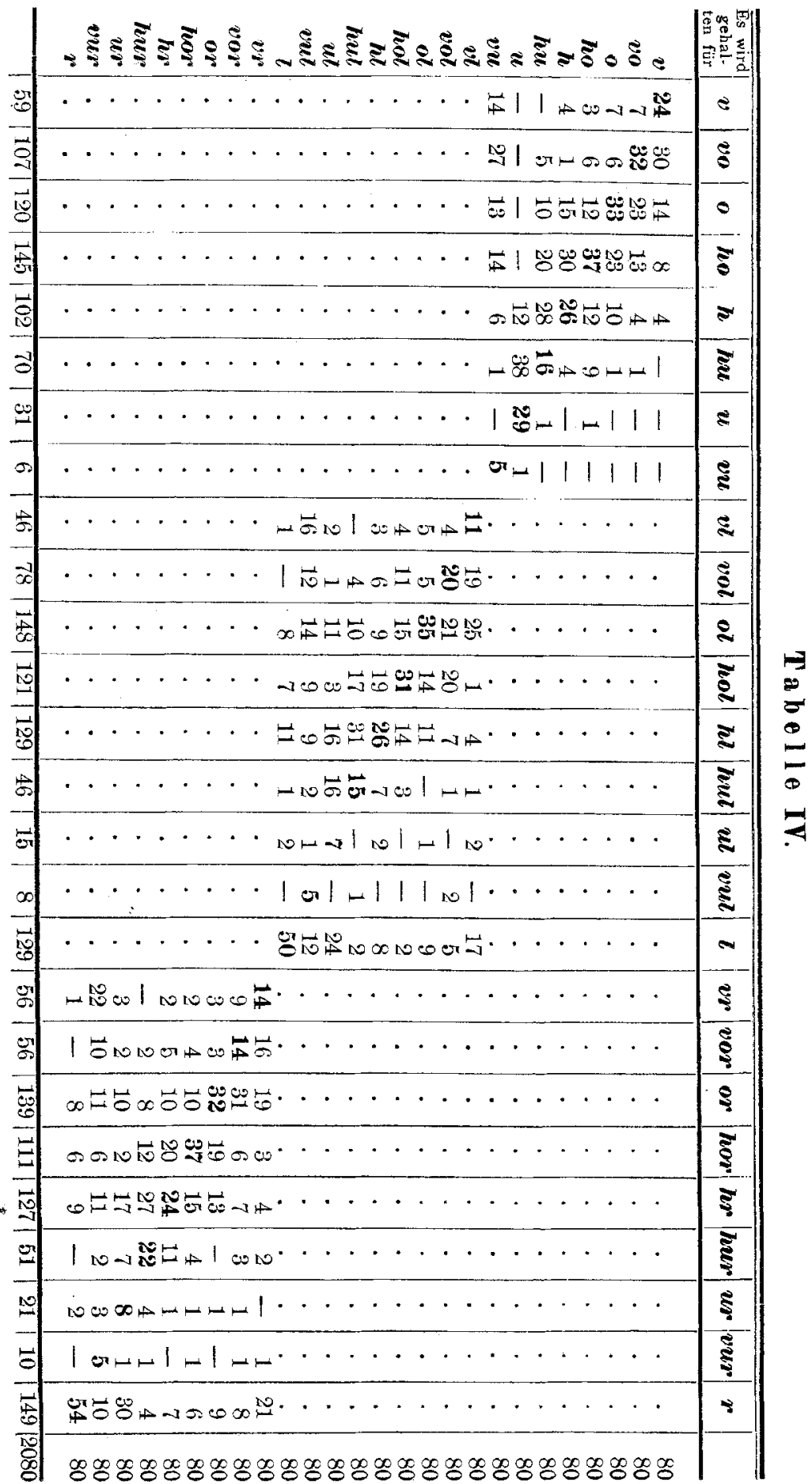


Die Wahrnehmung der Schallrichtang mittelst der Bogengänge. 605

Die richtigen Fälle sind selbstverständlich dieselben wie in der obigen Tabelle II (S. 601). Von den unrichtigen sind mehrere hervorzuheben, weil sie wiederum eine Bevorzugung gewisser Schallrichtungen vor anderen kennen lehren. Ich nenne sie kurz die Nullfälle. Es sind die folgenden.

Ke inmal wurde gehalten

$$
\begin{aligned}
& \text { o für vu; ol für vul; or für vur } \\
& h \text {,vu; } h l, v u l ; h r \text {, vur } \\
& h u \text { " } v \text {; hul "vl; hur "vr } \\
& l \text {,vol; } r \text { "vor } \\
& l \quad, v u l ; r \quad \text {, vur. }
\end{aligned}
$$

Die übrigen Nullfälle beziehen sich entweder nur auf die Richtungen in der Medianebene oder in der linken oder in der rechten Kopfhälfte, aber nicht auf alle drei, sie haben also nicht den Werth der vorstehenden. Es wurde gehalten keinmal oder nur einmal

$$
\begin{aligned}
& v \text { für } v u ; \quad v l \text { für } v u l ; \quad v r \text { für } \quad v u r(1) \\
& o \quad, u ; \quad \text { ol }, u l(1) ; \quad \text { or , ur (1) } \\
& \text { ho "vu; hol " hul; hor ,, vur (1) } \\
& \text { o , hu(1); ol ", hul; or , hur } \\
& \text { ho , u(1); hol "ul; hor ,ur(1) } \\
& \text { hu "vu; hul , vul(1); hur "vur (1) } \\
& u \text {, vu (1); ul , vul; ur , vur (1) } \\
& v \text {, hu; } \quad v l, h u l(1) \\
& v, u ; \quad . \quad \text { vr }, u r \\
& \text { vo "u; vol , ul; vor "ur (1) } \\
& \text { vo " vu; . . vor , vur }(1) \\
& h, u ; \quad . \quad \text {. } h r, u r(1) \\
& u, v \text {; } \\
& u, \text { vo; } \quad u l, \quad \operatorname{vol}(1) \\
& u, \quad o \text {; } \\
& u, h o \text {; } \\
& v u \quad \text {, } \quad \text {; } \quad v u l \quad, u l(1) \text {; }
\end{aligned}
$$

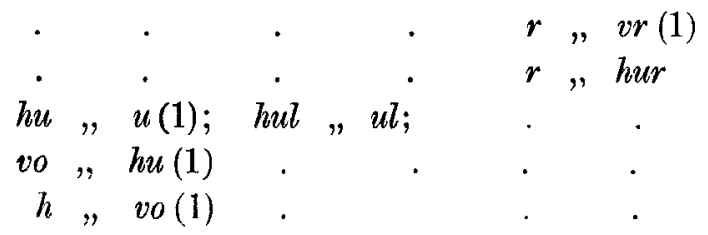


$v u$ für $h u(1)$

$$
\begin{gathered}
v l \text { für hol }(1) ; \\
\text { vol }, \operatorname{hul}(1) ; \\
l \quad, \operatorname{vl}(1) ; \\
l \quad, \operatorname{hul}(1) ;
\end{gathered}
$$

Diese 63 Verwechslungen (darunter 37 Nullfälle) gehören also zu den seltensten, welche iiberhaupt vorkommen.

\section{Die relative Frequenz der Schallrichtungsurtheile.}

Welche Schallrichtungen am häufigsten im Wahrnehmungsurtheil vorkommen, ergibt die unterste Zeile der letzten TabelleIV(S.604). Sie enthält die 26 Einzelsummen der sämmtlichen 2080 Urtheile.

Der Häufigkeit nach ordnen sie sich in folgende Reihen. Statt je 80 mal wurde geurtheilt

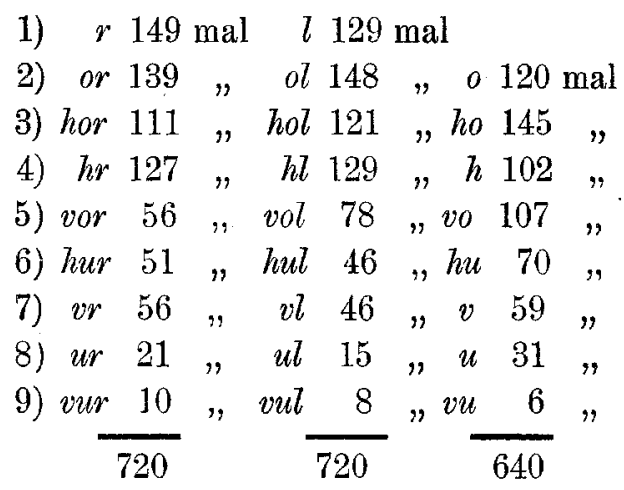

Von den 26 geprufften Schallrichtungen sind also $r$ und $l$, $o$ und $h$ und deren Combinationen viel öfter in der Wahrnehmung des Menschen als $v$ und $u$ und deren Combinationen.

Die eine Hälfte der 26 Kategorien bleibt unter der für jede geforderten Anzahl von 80 Urtheilen, die andere uibersteigt sie, und zwar um je 22 bis 69 bei $l, r, h, h l, h r, o, o l$, or, ho, hol, hor und $v o$, während das Deficit bei $u, u l, u r, v u, v u l, v u r, v, v l, v r, h u$, $h u l$, hur, vol und vor ebensoviel (im Ganzen 567) beträgt.

\section{Die Grösse der Fehler bezüglich ihrer Frequenz.}

Aus den obigen Gesetzmässigkeiten der Fehlervertheilung folgt schon rücksichtlich des Vorkommens der (S. 594) bereits zusammengestellten 12 verschiedenen Fehlergrössen, dass der grösste Fehler 
Die Wahrnehmung der Schallrichtung mittelst der Bogengänge. 607

von $180^{\circ}$ nur in der Medianebene vorkommen kann, was die Erfahrung bestätigt, da $l$ und $r$ nicht verwechselt werden. Aus demselben Grunde bleibt die Verwechslung zweier Schallrichtungen, welche einen Winkel von $1 \frac{13}{4}$ Rechten miteinander bilden, auf die Medianebene beschränkt.

Den kleinsten Fehlern von $35,3^{\circ}$ und $45^{\circ}$ kommen die meisten Fälle zu. Die folgende Tabelle lehrt das Weitere.

Tabelle V.

Bei Beurtheilung der Schallrichtungen betrug den Tabellen I und IV zufolge die Anzahl der Fehler für die einzelnen Winkel:

\begin{tabular}{r|r|r|r|r|r|r|r|r|r|r}
\hline $\begin{array}{r}\text { Rich- } \\
\text { tung. }\end{array}$ & $0^{0}$ & $35,3^{0}$ & $45^{0}$ & $54,7^{0}$ & $60^{0}$ & $70,0^{0}$ & $90^{0}$ & 109,50 & $135^{0}$ & $180^{0}$ \\
\hline $\boldsymbol{l}$ & 50 & - & 22 & 8 & - & - & - & - & - & - \\
$\boldsymbol{r}$ & 54 & - & 20 & 6 & - & - & - & - & - & - \\
\hline $\boldsymbol{h l}$ & 26 & 26 & 8 & - & 11 & - & 9 & - & - & - \\
$\boldsymbol{h r}$ & 24 & 31 & 7 & - & 11 & - & 7 & - & - & - \\
\hline $\boldsymbol{o l}$ & 35 & 19 & 9 & - & 16 & - & 1 & - & - & - \\
$\boldsymbol{o r}$ & 32 & 22 & 9 & - & 16 & - & 1 & - & - & - \\
\hline $\boldsymbol{v l}$ & 11 & 19 & 17 & - & 27 & - & 6 & - & - & - \\
$\boldsymbol{v r}$ & 14 & 17 & 21 & - & 19 & - & 9 & - & - & - \\
\hline $\boldsymbol{u l}$ & 7 & 16 & 24 & - & 18 & - & 15 & - & - & - \\
$\boldsymbol{u r}$ & 8 & 8 & 30 & - & 20 & - & 14 & - & - & - \\
\hline $\boldsymbol{h o l}$ & 31 & 29 & - & 2 & - & 14 & 4 & 0 & - & - \\
$\boldsymbol{h o r}$ & 37 & 25 & - & 6 & - & 8 & 3 & 1 & - & - \\
\hline $\boldsymbol{v o l}$ & 20 & 25 & - & 5 & - & 22 & 7 & 1 & - & - \\
$\boldsymbol{v o r}$ & 14 & 40 & - & 8 & - & 7 & 8 & 3 & - & - \\
\hline $\boldsymbol{h u l}$ & 15 & 31 & - & 2 & - & 18 & 10 & 4 & - & - \\
$\boldsymbol{h u r}$ & 22 & 31 & - & 4 & - & 13 & 8 & 2 & - & - \\
\hline $\boldsymbol{v u l}$ & 5 & 17 & - & 12 & - & 14 & 23 & 9 & - & - \\
$\boldsymbol{v} \boldsymbol{u r}$ & 5 & 25 & - & 10 & - & 12 & 22 & 6 & - & - \\
\hline $\boldsymbol{h o}$ & 37 & - & 24 & - & - & - & 15 & - & 4 & 0 \\
$\boldsymbol{o}$ & 33 & - & 29 & - & - & - & 17 & - & 1 & 0 \\
$\boldsymbol{v} \boldsymbol{o}$ & 32 & - & 30 & - & - & - & 13 & - & 4 & 1 \\
$\boldsymbol{v}$ & 24 & - & 30 & - & - & - & 14 & - & 8 & 4 \\
$\boldsymbol{u}$ & 29 & - & 39 & - & - & - & 12 & - & 0 & 0 \\
$\boldsymbol{h} \boldsymbol{h u}$ & 26 & - & 34 & - & - & - & 15 & - & 1 & 4 \\
$\boldsymbol{v} \boldsymbol{u}$ & 5 & - & 14 & - & - & - & 28 & - & 19 & 14 \\
\hline 2080 & 612 & 381 & 396 & 63 & 138 & 108 & 281 & 26 & 47 & 28 \\
& & - & 29 & - & - & - & 20 & - & 10 & 5 \\
\hline
\end{tabular}


Diese Tabelle bestätigt und erweitert wiederum frühere Ergebnisse. Namentlich zeigt sie den Parallelismus der Fehler links und rechts. Nicht nur die Anzahl, sondern auch die Grösse derselben weist auffallende Coüncidenzen auf.

Ausserdem erkennt man leicht, dass die kleinsten Fehler die häufigsten, aber durchans nicht in ihrer Frequenz etwa dem von der Wahrscheinlichkeitsrechnung geforderten für Beobachtungsfehler gültigen Gesetze der Vertheilung unterworfen sind.

Nach der oben (S. 594) gegebenen Zusammenstellung der denkbaren Fehler und der dann (S. 598) ermittelten empirischen Ausschliessungen grosser Gruppen derselben, bleiben nur 200 von den 650 tibrig, d. h. von 676 Fällen nur 226 , wenn man die 26 richtigen Fälle, als Fehler von $0^{0}$, mitzählt. Es kann nämlich, wie eine einfache Vergleichung der Tabellen I und IV zeigt, sich bei allen künftigen Bestimmungen nach der hier angewandten Methode nur um folgende Möglichkeiten handeln:

\section{Tabolle VI.}

\begin{tabular}{|c|c|c|c|}
\hline Fehler. & $\begin{array}{l}\text { Wie oft denkbar } \\
\text { möglich. }\end{array}$ & $\begin{array}{c}\text { Wie oft empirisch } \\
\text { möglich. }\end{array}$ & $\begin{array}{c}\text { Wie oft } \\
\text { beobachtet. }\end{array}$ \\
\hline 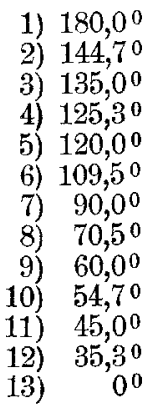 & $\begin{array}{r}26 \\
48 \\
48 \\
48 \\
48 \\
24 \\
192 \\
24 \\
48 \\
48 \\
48 \\
48 \\
26\end{array}$ & 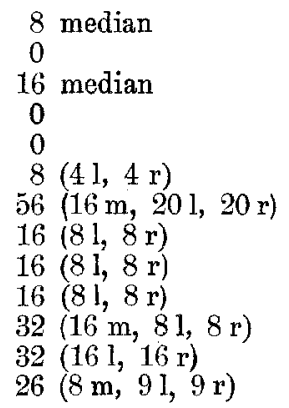 & $\begin{array}{l}\bar{\partial} \text { median } \\
\overline{9} \text { median } \\
- \\
\overline{7} \\
41(31,4 \mathrm{r}) \\
16(11 \mathrm{~m}, 141,16 \mathrm{r}) \\
15(81,7 \mathrm{r}) \\
11(6 \mathrm{l}, 5 \mathrm{r}) \\
30(14 \mathrm{~m}, 8 \mathrm{l}, 8 \mathrm{r}) \\
29(13 \mathrm{l}, 16 \mathrm{r}) \\
26(8 \mathrm{~m}, 9 \mathrm{l}, 9 \mathrm{r})\end{array}$ \\
\hline & 676 & 226 & 189 \\
\hline
\end{tabular}

Die Differenz 226-189 = 37 entspricht den empirisch möglichen, aber bis jetzt nicht beobachteten, nur sehr selten, zum Theil vielleicht gar nicht vork ommenden Fällen von Richtungsverwechslungen, d. h. den 37 Nullfällen der Tabelle IV (S. 604). Den eingeklammerten Ziffern zufolge ist es jedoch sehr wahrscheinlich, 
dass wenigstens 8 von diesen Nullfällen zufällig sind, wegen der sonstigen Uebereinstimmung von $l$ und $r$.

Dass die Winkel 2) und 4), sowie 5) nicht bei normal Hörenden vorkommen können, ist durch das streng gültige einseitige Localisiren auf der linken oder auf der rechten Kopfhälfte ohne Kreuzung bedingt (S. 597). Aus eben diesem Grunde sind die grossen Fehler 1) und 3) auf die Medianebene beschränkt, wie bereits hervorgehoben wurde (S. 607).

Um schliesslich die beobachteten Fehlersummen für die einzelnen Winkel mit den möglichen zu vergleichen, dient die folgende Zusammenstellung, in der nur die empirisch möglichen, d. h. die mit Ausschluss der normalerweise nicht vorkommenden gekreuzten und $l$ und $r$-medianen Fehler (S. 597) noch möglichen nach ihrer Häufigkeit aufgenommen sind.

Bei 80-maliger Beurtheilung jeder der 26 Richtungen kommen bei der Vertheilung der Fehlerwinkel nach ihrer Häufigkeit (S. 594, 607 und 608) auf

\begin{tabular}{cr|r|r|r|r|r|r|r|r|r|r|r|r}
\hline & $0^{0}$ & $35,3^{0}$ & $45^{0}$ & $54,7^{0}$ & $60^{\circ}$ & $70,5^{0}$ & $90^{0}$ & 109,50 & $135^{0}$ & $180^{0}$ & \\
\hline Fälle & 612 & 381 & 396 & 63 & 138 & 108 & 281 & 26 & 47 & 28 & 2080 \\
statt 392 & 241 & 271 & 121 & 121 & 121 & 392 & 121 & 150 & 150 & 2080 \\
\hline Diff. +220 & +140 & +125 & -58 & +17 & -13 & -111 & -95 & -103 & -122 &
\end{tabular}

Es muss also im Gehörorgan eine Eiurichtung bestehen, welche die auffallenden Differenzen verursacht. Diese Differenzen fallen natïrlich noeh viel grösser aus, wenn man die überhaupt (mathematisch) möglichen Fälle, ohne Ausschluss der gekreuzten und der l. und $r$-medianen Fehler (S. 597) mit den beobachteten vergleicht.

\section{Die specifische Lnergie der Ampullennerven.}

Wenn man bedenkt, dass die Fische, welche keine Schnecke haben, nicht allein hören, sondern auch trotz Fehlens einer äusseren Ohröffnung, die Richtung erkennen, aus welcher ein Schall kommt, so erscheint die Vermuthung gerechtfertigt, dass es die Bogengänge seien, welche mittelst Kopfleitung diese Leistung ermöglichen, indem, je nach der Richtung des Schallstrahls in Bezug auf das Thier, der eine oder der andere Bogengang stark, bald zwei oder drei der sechs Ampullen, bald keine derselben, hinreichend stark afficirt 
werde, um eine Schallempfindung mit dazugehörigem Raumgefühl zu Stande kommen zu lassen.

Die Kopfleitung ist eine Thatsache und kommt beim Hören der ausschliesslich im Wasser lebenden Wirbelthiere allein in Betracht. Diese Fortpflanzung des Schalles ist sogar bei den Fischen wegen des Wasserreichthums ihres Kopfes ohne Zweifel begïnstigt im Vergleiche zu derselben bei den übrigen nur temporär oder garnicht im Wasser verharrenden Wirbelthieren.

Es lässt sich aber nicht leugnen, dass durch Schallstrahlen verschiedener Einfallsrichtung in jedem Wirbelthierkopfe, wie im Fischkopfe, die Endolymphe in den häutigen Bogengängen in Erschütterungen von derselben Frequenz und entsprechender Stärke versetzt werden muss, wie sie das Wasser oder die Luft ausserhalb erfährt. Dann werden diese aber auch wegen der an jedem Puncte jedes Bogenganges vorhandenen Krïmmung sofort die ganze Masse der Flüssigkeit in isochrone Bewegungen versetzen müssen. Hierdurch kommen die in den Ampullen in die Endolymphe hineinragenden Hörhaare nothwendig in Mitschwingungen, und wenn man erwägt, dass bei derartiger Erregung einer Ampulle durch Erschütterung der Flüssigkeit nur des ihr zugehörigen Bogenganges eine andere Schallempfindung bei gleicher Stärke, Tonhöhe und Klangfarbe, als bei Erregung einer anderen Ampulle entstehen muss (da es ja andere Nervenfasern ${ }^{1}$ ) sind, die erregt werden), so wird man diese Verschiedenheit nicht anders, denn als eine räumliche bezeichnen können. Das Thier hat seit unzählbaren Generationen ausnahmslos, wenn die Nerven der Ampulle I links stärker erregt waren, als die der anderen Ampullen, auch als die von II und III links, die Erfahrung gemacht, dass ein Schall gerade von links ber kam und so

1) „Die Menschen besitzen für die Raumwahrnehmung kein besonderes äusseres Sinnesorgan, vielmehr vermittelt sie jeder der fünf sinne. Indem gleichartige Erregungen verschiedener Nervenfasern, welche gleiche Empfindungen geben, doch unterschieden werden, kommen Raumvorstellungen zu Stande. Aus den Beziehungen der erregten Nervenfasern zu einander schliesst der Verstand, auch ohne sie zu kennen, unbewusst, dass das Gefühlte, das Gesehene den erregten Nervenenden entsprechend, nebeneinander, übereinander, hintereinander liege. Und indem eine einzelne Nervenfaser gleichartige Empfindungen gibt, welche dennoch unterschieden werden, kommen zeitliche Wahrnehmungen zu Stande." (Preyer, „Die fünf Sinne des Menschen̈", 1870, S. 41). 
für die anderen Ampullen, die dem Oben (II) und Hinten (III) entsprechen.

Es ist also eine völlig legitime Hypothese, wenn ich behaupte: die specifische Energie der Ampullennerven ist es, ein mit Schall verbundenes Raumgefühl zu geben, und zwar ein Richtungsgefühl. Die Art dieses Eindrucks ist verschieden je nach der Richtung, ans welcher der Schall herkommt und wird durch diesen bestimmt, indem die einzelnen Schallrichtungen, welche überhaupt erkannt werden können, immer einen Bogengang oder ein Bogengangpaar stärker als die anderen treffen miissen.

Ich nehme an, dass die sechs Bogengänge folgende specifische Energien haben.

Horizontaler Bogengang links (Il). Er wird am stärksten erregt bei den Schallrichtungen von links her in der horizontalen Ebene.

Horizontaler Bogengang rechts (I r). Ebenso von rechts her.

Oberer verticaler (vorderer) Bogengang links (II I). Er wird am stärksten erregt bei den von oben und von oben und vorn kommenden Schalleindrücken, welche die linke Kopfhälfte mehr als die rechte treffen.

Oberer verticaler (vorderer) Bogengang rechts (II r). Er wird am stärksten erregt bei den von oben und vorn-oben kommenden Schalleindrücken, welche die rechte Kopf'bälfte mehr als die linke treffen.

Unterer verticaler (hinterer) Bogengang links (III l). Er wird am stärksten erregt bei den von hinten und von hintenunten kommenden Schalleindrücken, welche die linke Kopfhälfte mehr als die rechte treffen.

Untererverticaler (hinterer) Bogengang rechts (IIIr). Desgleichen; nur ist statt links zu setzen rechts und umgekehrt.

Dieses einfache Princip genügt, um fast alle in dieser Arbeit gewonnenen Thatsachen in einen natürlichen Zusammenhang zu bringen und die neu gefundenen Gesetze der Verwechslungen verständlich zu machen.

Ich setze dabei selbstverständlich voraus, dass beim normalen Hören mittelst der Kopfleitung ebenso wie mittelst der Luftleitung bei nngleich starker Erregung beider Ohren nur eine Schallwahrnehmung gemacht, aber die Schallquelle nach der Seite der stär- 
keren Erregung verlegt wird („Princip der Verlegung des Schalles nach der Seite der stärkeren Erregung" Kessel, im Archiv für Ohrenheilkunde 18. Bd. S. 121. 1882, u. Chladni, Akustik, 1802. 250).

Die höheren Wirbelthiere, von den Fischen aufwärts, hören sämmtlich, ausser durch Luftleitung, auch durch Kopfleitung, haben sämmtlich wohl ausgebildete Bogengänge in ähnlicher Anordnung, wie aus dem monumentalen Werke über das Gebörorgan der Wirbelthiere von Gustav Retzius (Stockholm 1881 und 1884) leicht zu ersehen, und alle können die Schallrichtung erkennen. Es liegt kein Grund vor, ihren Bogengängen eine andere Function zuzuschreiben, als denen der Fische. Ich stehe nicht an, auch für den Menschen dieselbe specitische Energie der Ampullennerven zu behaupten, und stelle hier zunächst die Vertheilung der 26 Schallrichtungen auf die 6 Bogengänge zusammen, indem ich nur noch vorher wiederhole, dass es die ungleiche Intensität der Schalleindrücke im Kopfe und damit der Schallempfindungen ist, welche bestimmt, ob die letzteren nach links oder rechts verlegt werden. Ist nämlich die Intensität beiderseits dieselbe, dann hört man weder links noch rechts, sondern verlegt den Schallort in die Medianebene des Kopfes, was längst bekannt ist.

Die 26 untersuchten Schallrichtungen zerfallen hiernach von selbst in die 3 schon oben unterschiedenen Gruppen.

I 1 und I $\mathrm{r}$ bezeichnet die beiden borizontalen, II 1 und II $r$ die beiden verticalen, oberen, vorderen, III 1 und III $r$ die beiden verticalen, unteren, binteren Bogengänge.

Von der Voraussetzung ausgehend, dass bei jedem Schall die Nerven aller sechs Ampullen erregt, aber in ungleichem Verbältniss, d. h. in verschiedener Stärke erregt werden, je nach dem Winkel, welchen ibre Ebene mit der Schallriehtung bildet und je nachdem sie der Schallquelle näher oder ferner liegen, nenne ich in der folgenden Tabelle:

1 die relativ schwächste Erregung;

2 die mittelstarke Erregung,

3 die relativ stärkste Erregung,

und beziehe diese Ziffern sowohl auf die durch Kopfleitung, als auch auf die durch Luftleitung des Schalles verursachte Bewegung der Labyrinthflïssigkeit beim gewöhnlichen Hören. 
Tabelle VII.

\begin{tabular}{|c|c|c|c|c|c|c|c|}
\hline \multirow{2}{*}{\multicolumn{2}{|c|}{ Schallrichtung. }} & \multicolumn{6}{|c|}{ Erregung der Ampullennerven. } \\
\hline & & II. & II 1 & III 1. & I $\mathbf{r}$. & II $r$ & III $r$. \\
\hline 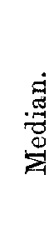 & $\begin{array}{l}\boldsymbol{v} \\
v o \\
o \\
h o \\
h \\
h u \\
u \\
v u\end{array}$ & $\begin{array}{l}2 \\
2 \\
2 \\
2 \\
2 \\
2 \\
2 \\
2\end{array}$ & $\begin{array}{l}3 \\
3 \\
3 \\
2 \\
1 \\
1 \\
1 \\
2\end{array}$ & $\begin{array}{l}1 \\
1 \\
1 \\
3 \\
3 \\
3 \\
3 \\
2\end{array}$ & $\begin{array}{l}2 \\
2 \\
2 . \\
2 \\
2 \\
2 \\
2 \\
2\end{array}$ & $\begin{array}{l}3 \\
3 \\
3 \\
2 \\
1 \\
1 \\
1 \\
2\end{array}$ & $\begin{array}{l}1 \\
1 \\
1 \\
3 \\
3 \\
3 \\
3 \\
2\end{array}$ \\
\hline $\begin{array}{l}\dot{9} \\
\dot{M} \\
\dot{A}\end{array}$ & $\begin{array}{l}l \\
v l \\
v o l \\
o l \\
h o l \\
h l \\
h u l \\
u l \\
v u l\end{array}$ & $\begin{array}{l}3 \\
3 \\
3 \\
3 \\
3 \\
3 \\
3 \\
3 \\
3\end{array}$ & $\begin{array}{l}1 \\
3 \\
3 \\
3 \\
2 \\
1 \\
1 \\
1 \\
2\end{array}$ & $\begin{array}{l}1 \\
1 \\
1 \\
1 \\
3 \\
3 \\
3 \\
2 \\
2\end{array}$ & $\begin{array}{l}1 \\
1 \\
1 \\
1 \\
1 \\
1 \\
1 \\
1 \\
1 .\end{array}$ & $\begin{array}{l}1 \\
1 \\
1 \\
1 \\
1 \\
1 \\
1 \\
1 \\
1\end{array}$ & $\begin{array}{l}1 \\
1 \\
1 \\
1 \\
1 \\
1 \\
1 \\
1 \\
1\end{array}$ \\
\hline 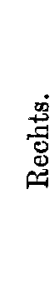 & $\begin{array}{l}\boldsymbol{r} \\
\boldsymbol{v r} \\
\text { vor } \\
\text { or } \\
\boldsymbol{h o r} \\
\boldsymbol{h r} \\
\boldsymbol{h u r} \\
\boldsymbol{u} \boldsymbol{u r} \\
\text { vur }\end{array}$ & $\begin{array}{l}1 \\
1 \\
1 \\
1 \\
1 \\
1 \\
1 \\
1 \\
1\end{array}$ & $\begin{array}{l}1 \\
1 \\
1 \\
1 \\
1 \\
1 \\
1 \\
1 \\
1\end{array}$ & $\begin{array}{l}1 \\
1 \\
1 \\
1 \\
1 \\
1 \\
1 \\
1 \\
1\end{array}$ & $\begin{array}{l}3 \\
3 \\
3 \\
3 \\
3 \\
3 \\
3 \\
3 \\
3\end{array}$ & $\begin{array}{l}1 \\
3 \\
3 \\
3 \\
2 \\
1 \\
1 \\
1 \\
2\end{array}$ & $\begin{array}{l}1 \\
1 \\
1 \\
1 \\
3 \\
3 \\
3 \\
2 \\
2\end{array}$ \\
\hline
\end{tabular}

Wenn diese Hypothese richtig ist, dann muissen alle diejenigen Schallrichtungen, denen annähernd gleiches Erregungsstärkeverhältniss der Ampullennerven entspricht, besonders häufig miteinander verwechselt werden, und sie werden überhaupt dann garnicht voneinander durch das Gehör allein unterschieden werden können, wenn ihre relativen Erregungsgrade genau gleich sind. Für die Richtungsverwechslungen

$$
\begin{aligned}
& v \text {-vo, } h-h u, h u-u, \\
& \text { vol-ol, vl-ol, vl-vol, } h l-h u l, \\
& \text { vor-or, vr-or, vr-vor, hur-hr }
\end{aligned}
$$

müsste also diese Consequenz vor allem zutreffen. Ein Blick auf S. 603 und die Zahlenbelege Tabelle II-IV zeigt, dass es wirklich der Fall ist. Aber auch die Verwechslungen

$$
v-o, v o-o, u l-h u l, u r-h u r \text {, }
$$

sind von einer so grossen Häufigkeit, dass sie in dieselbe Kate- 
gorie gestellt werden mïssen, und dass die Verwechslung $h-u$ nicht so oft vorgekommen ist, wie hiernach zu erwarten, liegt ohne Zweifel an der geringeren Schwierigkeit den Schallort unten (unter dem Stuhle) wegen der grösseren Distanz vom Ohre zu erkennen, denn die Verwechslungen hl-ul, hr-ur sind häufig (S. 601).

Ferner müssen sehr häufig verwechselt werden wegen Aehnlichkeit der Erregungsrelationen:

$$
\text { ho-h, hol-hl, hor-hr, ho-hu, hol-hul, hor-hur }
$$

was auch die Erfahrung bestätigt. Dass die Verwechslung ho-u sehr selten vorkommt, ist wahrscheinlich durch die abweichende Lage des $u$ bedingt, die grosse Leichtigkeit der Verwechslungen $u l-l$ aud $u r-r$, in der Tabelle II hingegen vorgesehen.

Nur sehr selten, bei höchster Anspannung der Aufmerksamkeit vielleicht garnicht, werden ferner verwechselt werden dürfen

$$
\begin{aligned}
& \text { v-u, vl-ul, vr-ur, } \\
& \text { v-hu, vl-hul, vr-hur, hol-ul } \\
& \text { vo-u, vol-ul, vor-ur, hor-ur }
\end{aligned}
$$

was die Versuche beweisen (\$. 605).

Dasselbe gilt wahrscheinlich für

$$
\begin{aligned}
& v-h o, v l-h o l, v r-h o r \\
& v-h, v l-h l, v r-h r \\
& \text { vo-hu, vol-hul, vor-hur } \\
& \text { vo-h, vol-hl, vor-hr } \\
& \text { o-hu, ol-hul, or-hur } \\
& \text { o-u, ol-ul, or-ur }
\end{aligned}
$$

welche aber (Tab. III) nicht ganz so selten miteinander verwechselt worden sind, wie die vorigen. Viele Versuche an einem und demselben Individuum werden zeigen, ob die Anzahl der Fehler nicht schliesslich sehr klein wird.

Am genauesten wird stets, durch das Gehör allein, von den medianen Schallrichtungen ho erkannt werden müssen, weil es allein das Erregungsverhältniss 2, 2, 3 beiderseits hat. In der That ist die Anzahl der richtigen Fälle für ho ein Maximum. Von allen Richtungen überhaupt aber werden $l$ und $r$, die mit den Höraxen zusammenfallen und die grösste Intensität haben, am besten beurtheilt werden müssen, weil sie allein das Verhältniss $3,1,1$ haben. Die Verwechslungen von $l$ mit $v l$, vol, ol, hol, $h l$, $h u l$, bez. von $r$ mit $v r$, vor, or, hor, hr, hur, können sämmtlich auf Nachlässigkeit, $d$. h. auf Schätzungsfehlern beruhen und bediirfen keiner 
Herbeiziehung organischer Mängel. Denn die grössten Fehler betragen nur $45^{\circ}$ bis höchstens $55^{\circ}$ (Tab. V) und werden meist bei Wiederholung der Prïfung und Vergleichung mit dem wahren $l$ und $r$ schnell corrigirt. Uebrigens entspricht die Häufigkeit dieser Verwechslungen bei Ungeübten durchaus der Tabelle VII.

Bezüglich der Erklärung der übrigen Verwechslungen sei folgendes bemerkt.

$$
\begin{aligned}
& \text { ho-o, hol-ol, hor-or } \\
& \text { ho-vo, hol-vol, hor-vor } \\
& \text { h-o, hl-ol, ho-ol }
\end{aligned}
$$

werden immer dann zu den bäufigeren Verwechslungen gehören, wenn auf den Schallort nicht genau eingestellt wurde, z. B. ho etwas näher bei $o$ als bei $h$ zu liegen kam, weil dann die Erregung des vorderen-oberen Bogenganges (II) im Verhältniss zu der des hinteren-unteren (III) zunehmen muss, wodurch, da letztere dann absolut und relativ abnimmt, eine starke Annäherung an die für o charakteristische Erregungsvertheilung herbeigeführt wird. Diese Aenderung ist bei den Versuchen anfangs nicht selten unabsichtlich vorgekommen. Doch erkennt man aus der Tabelle IV, dass alle 9 Verwechslungen (ausser 0 -ho) nicht zu den häufigsten gehören, entsprechend den ungleichen Erregungsverhältnissen.

Eine besondere Erörterung verlangt die Beurtheilung der Scballrichtung $v u$, nebst vul und vur. Die Anzahl der richtigen Fälle ist hier minimal, die Mannigfaltigkeit und Grösse der Fehler maximal (Tab. IV, V). Unter allen Schallrichtungen wird $v u$ bei weitem am schwierigsten durch das Gehör allein erkannt. Ich habe geglaubt diese Thatsache für $v u$ in der Tabelle VII dadurch ausdrücken zu sollen, dass ich allen 6 Ampullen die gleiche Erregungsgrösse zuschrieb. Dann ist der Beurtheilende in Verlegenheit, woher der Schall kommt und verlegt ihn in mehreren aufeinanderfolgenden Versuchen fast jedesmal an einen anderen Ort in der Medianebene.

Dass normaler Weise eine Verwechslung irgend einer Schallrichtung, die irgendwo die linke Kopfbälfte trifft, mit einer, welehe die rechte trifft, nicht vorkommt, erklärt sich einfach durch die sehr grosse Verschiedenheit der Erregungsgrade und deren ungleiche Vertheilung auf die 3 Bogengänge links und rechts. (Verlegung auf die Seite der stärkeren Erregung.)

Für die Thatsache, dass normaler Weise kein von links oder 
von rechts kommender Schall in die Medianebene verlegt wird, und für die andere Thatsache, dass keine mit einem Radius der Medianebene zusammenfallende Schallrichtung nach links oder nach rechts verlegt wird, gilt dasselbe. Alle diese factisch durch die mitgetheilten Versuche bewiesenen Gesetzmässigkeiten erscheinen auf Grund meiner Hypothese verständlich. Eine graphische Darstellung ähnlich derjenigen, welche von Maxwell 1860 zur Erklärung der Farbenverwechslungen Farbenblinder (Pflü ge r's Archiv XXX, Taf. II, Fig. 7 u. a.) entworfen wurde, d. h. superponirte Curven, deren Ordinaten die Erregungsgrade der Ampullennerven und deren Abscissen die Schallrichtungen schematisch angeben, veranschaulicht die Gesammtheit der Verwechslungen der letzteren auf das Beste. Doch sehe ich hier davon ab, weil der Gegenstand schon genügend deutlich gemacht ist.

Hingegen war es erforderlich, um die Voraussetzung von der Betbeiligung der Kopfleitung an der Localisation der Schalleindrïcke an der Erfahrung zu prüfen, dass Versuche mit möglichstem Ausschluss der Luftleitung angestellt wurden, dass auch das Localisiren bei einseitiger Taubheit und schliesslich mit möglichst weitgehender Verminderung der Kopfleitung und erhaltener Luftleitung experimentell gepriift wurde.

\section{Die Frkennung der Schallrichtung mit beiderseits verschlossenen Ohren.}

Ein einfaches Mittel, vorïbergehend beiderseits die Luftleitung auszuschliessen, ist der Verschluss beider Ohren mit je einem Finger, welcher entweder in den äusseren Gebörgang eingeführt wird oder durch festes Andrïcken des umgelegten Tragus die Luft absperrt. Um aber eine etwaige Betheiligung der Ohrmuschel zu eliminiren, haben wir auch in einigen Versuchsreihen diese ganz mit Glaserkitt bedeckt und an den Kopf gekittet. Um endlich die Luft im äusseren Gehörgange ganz zu entfernen, haben wir den letzteren mit lawwarmer physiologischer Kochsalzläsung angefüllt und dann mit dem Finger zugehalten. Da aber die letztgenannten Verschlussarten sehr unangenebm sind, subjectiver Schall nachher noch lange im Ohre verharren kann, wenn das Wasser länger mit dem Trommelfell in Berihhrung bleibt, und die Resultate aller dieser Versuche vollkommen mit der vorgetragenen Erklärung überein- 
stimmen, so haben wir nicht viel' mehr als dreibundert Einzelbestimmungen protokollirt. Es ergibt sich ans diesen folgendes.

1) Die Bevorzugung der Schallrichtungen links und rechts im Vergleich zu allen anderen, fällt nach Ausschluss der Luftleitung: fort oder tritt zurïck.

Diese Thatsache erscheint natürlich, weil beim gewöhnlichen Hören in der Luft die beiden horizontalen Bogengänge mit den specifischen Energieen Schall lintes und Schall rechts bei den Excursionen der Fussplatte des Steigbügels viel leichter als die vier anderen afficirt werden mïssen; denn der Steigbügel liegt in einer zum horizontalen Bogengang fast genan parallelen Ebene, wie ich mich bestimmt an den vorzitglichen von Hrn. Dr. Götze hier angefertigten Metallausgiissen des menschlichen Labyrinths - auch vom Neugeborenen - überzeugt habe. Man sieht an diesen besser als an irgend einem anatomischen Präparat den Parallelismus. Fällt die Luftleitung fort, werden also die Trommelfell- und Steigbuigelschwingungen enorm vermindert, dann muss auch jener beim Hören in der Luft so auffallende Vortheil fortfallen, welehen die horizontalen Bogengänge vor den anderen voraus haben.

2) Verlegungen von Schallrichtungen aus der Medianebene nach links und rechts, von links und rechts in die Medianebene, sogar von links nach rechts und umgekehrt, kommen zwar, wie beim Hören mit offenen Ohren, beim erstmaligen Prüfen ror, werden aber meistens schnell auch von dem Ungeübten corrigirt; und da gate Beobachter, namentlich Hr. K. Schäfer selbst, welcher sehr schnell und sicher die Sehallichtungen angibt, ganze Reihen obne einen einzigen derartigen Fehler liefern, so kann darin eine Verschiedenheit des Hörens mittelst der Kopfleitung allein im Vergleiche zu dem gewöhnlichen Hören nicht gefunden werden. Nicht selten hat das Versuchsindividuum sich nur versprochen.

3) Aber die Erkennung der Sehallrichtung ist nach Verschluss beider Ohren erbeblich erschwert, das Urtheilen oft verzögert. Dieser Unterschied erklärt sich durch die absolute Intensitätsabnahme der Schallempfindung und die dadurch bedingte absolute Abnahme der Intensitätsdifferenzen, welche die Beurtbeilung der relativen Erregungsgrössen erschwert.

4) Daher ist überhaupt die Anzahl der genau richtigen Urtheile beim Hören mit fest verschlossenen Ohren etwas geringer, 
als beim gewöhnlichen Hören in der Luft. Doch waren in einzelnen Reihen $30 \%$ völlig richtig, während es vorgekommen ist, dass ein normalhörender Student mit offenen Ohren unter sonst gleichen Umständen $20 \%$ nicht erreichte.

Es wäre wichtig solche Versuche an Individuen anzustellen, welche beiderseits für die Schallschwingungen der Luft dureh pathologische Veränderungen des Mittelohrs unempfindlich geworden sind, aber noeh mittelst der Kopfleitung hören können.

\section{Die Erkennung der Schallichtung nach Verschluss eines Ohres.}

Um bei der Beurtheilung einer Scballrichtung mittelst eines Ohres sicher zu sein, dass das andere nicht im geringsten mitwirkt, verfiel ich darauf den Schall des Cri-cri durch Entfernung von der Schallquelle bis zur Unhörbarkeit abzuschwächen, während beide Ohren festgeschlossen gehalten wurden; dann musste, wenn nun ein $\mathrm{Ohr}$ geöffnet ward, der ferne Schall nur durch dieses wahrgenommen werden. Ich gab jedoch dieses Verfabren bald wieder auf, weil die Entfernungen zu gross genommen werden mussten. Hr. Schäfer hat die Methode wieder aufgenommen und sehr brauchbar gemacht, indem er bei demselben Abstande vom Kopfe, wie bisher, das Oeffnungsticken des Telephons mittelst Verminderung der Stromstärke so schwächte, dass es bei beiderseits geschlossenen Obren nicht erkannt wurde. Nach Oeffnen eines Ohres wurde es dann nur dureh dieses gehört, zwar ausschliesslich durch Luftleitung (vgl. jedoch S. 620 oben), aber deutlich genug, um beurtheilt zu werden.

Hierbei zeigte sich, dass die drei Classen von Fehlern, welehe bisher nicht als organische bezeichnet werden durften, sondern allemal corrigirt wurden, sich häufen und nicht mehr durch noch so angespannte Aufmerksamkeit zu beseitigen waren, nämlich die gekreuzten Urtheile, die Verlegungen von $l$ und $r$ in die Medianebene und umgekehrt. War das rechte Ohr mit Salzwasser verschlossen, so wurde von mir selbst nicht allein eine sehr starke Neigung verspürt fast jeden Schall nach links zu verlegen, sondern auch in acht Fällen fünfmal thatsächlich ein von $r$ kommender Schall unverbesserlich nach $l$ verlegt. Andere begingen derartige Fehler selten oder gar nicht. Immerhin ist es bemerkenswerth, dass die im gewöhnlichen Leben von einseitig tauben oder einseitig 
schwerhörigen Menschen gemachten Erfahrungen sich hier auch bei Prüfung der besten Beobachter bestätigt finden. Schon 1802 sprach Chladni den Satz aus, wem das eine Ohr verstopft sei, so scheine der Schall immer von der Seite des offenen Ohres (bei verbundenen Augen in gleicher Stellung) herzukommen, freilich ohne ihn durch Versuche zu begriunden. Er ist für keinen Mensehen vollkommen richtig, welcher mit dem Cri-cri geprifft wird, trifft aber in einzelnen Reiben zu.

Wenn man bedenkt, dass durch Ausschaltung eines Ohres die Erregungsverhältnisse der Tabelle VII gänzlich verändert werden, so dass bei dem leisen Schall, dessen Richtung beurtheilt werden soll, die Ampullennerven der einen Seite entweder garnicht oder nur minimal erregt werden, so erscheint es begreiflich, dass die des anderen ihre Energie in der Mehrzahl der Proben fast allein zur Bethätigung bringen, daher nicht nur mehr, sondern auch andere Fehler als normalerweise zu Stande kommen.

Umfassende Beobachtungsreiben an einseitig total Tauben wären zur genaneren Feststellung derselben sehr wünschenswerth.

\section{Die Erkennung der Schallrichtung nach Ausschluss der Kopfleitung.}

Die Ansicht scheint ziemlich allgemein verbreitet zu sein, dass beim gewöhnlichen Hören, z. B. gesprochener Worte, der Schall nur durch die Luft dem Labyrinthwasser zugefuibrt werde. Ibre Unrichtigkeit beweist schon die auch Chladni bekannte Thatsache, dass man nach Verschluss beider Ohren noch sehr viel hïrt and viele gesprochene Wörter versteht. Durch mündliche Mittheilungen des Hrn. Prof. Kessel hier über das Hören Ohrenkranker bin ich darin bestärkt worden, dass beim gewöhnlichen Hören mit offenen Ohren die Kopfleitung eine sehr wichtige Rolle spielt und ohne sie die Localisirung des Schalles noch viel mangelhafter sein würde, als sie ist. Die ganze vorstehende Untersuchung hat diese Voraussetzung begünstigt. Ich wünschte sie jedoch unmittelbar durch Ausschluss oder sehr weitgehende Verminderung der Kopfleitung zu beweisen.

Eine völlige Ausschliessung der Leitung durch die Kopftheile lässt sich jedoch schwerlich erzielen. Ich habe durch Abschwä. chung des Telephontons bis zur Unhörbarkeit bei verseblossenen Obren und dann noch etwas weiter getriebene Verminderung der 
erreichten Strom- und damit Schall-Stärke einen so schwachen Schallreiz herstellen können, dass auch bei unvollständigem Verschluss beider Obren nichts gehört wurde. Wurde er also mit ganz offenen Ohren gehört, so wurde er nur durch Luftleitung gehört, wenn nicht die Kopfleitung vom offenen Gehörgang aus erheblich ginstiger als von irgend einem anderen Punkte aus ist, was sich als sehr wahrscheinlich darthun lässt. Daher diese Versuche die Kopfleitung nur vermindern, nicht ausschliessen.

Es hat sich aber dabei herausgestellt, dass die Beurtheilung der Schallrichtung, wenn die Schallstärke so gering geworden ist, ausserordentlich erschwert und das Urtheilen anffallend unsicher wird, wenn auch die Anzahl der völlig richtigen Fälle wahrscheinlich zufällig nicht in jeder Reihe stark abnimmt. Nichts spricht aber gegen die vorgetragene Erklärung.

Dass von 132 Urtheilen von 3 Individuen 37 also $36,7 \%$ richtig waren, beweist nichts dagegen, weil 2 davon die geiibtesten waren (Hr. Schäfer und ich selbst) und bei so geringer Schallstärke die Aufmerksamkeit enorm angespannt wird. Der Ungeübte erkannte von den 26 Schallrichtungen der Sehallhaube nur 5, also $19,2 \%$ und zwar nicht sicher. Uebrigens wurden fast alle 132 Urtheile zögernd und zweifelnd abgegeben. Die Anzahl der Fälle ist zu klein, um den Zufall zu beherrsehen.

\section{Der Einfluss der Uebung.}

Dass durch häufige Wiederholung der beschriebenen Versuche die Sicherheit in der Beurtheilung der Schallrichtungen zunimmt, ist höchst wahrscheinlich, ich würde sagen gewiss, wenn nicht zum Beweise einer solchen Behauptung viel längere Versuchsreihen an einem Individuum nöthig wären, als bis jetzt vorliegen. Die durch häufige Wiederholung der Hörproben herbeigeführte Ermüdung und damit zusammengehende Unaufmerksamkeit der meisten unbefangenen Beurtheiler veranlasste uns, solche Versuchsreihen einstweilen nicht auszuführen. Andererseits mussten wir von uns selbst als Versuchsindividuen nach dieser Richtung absehen, weil durch zu viele Proben leicht ein Zustand der Befangenheit durch starke Anspannung der Aufmerksamkeit und den lebhafter werdenden Wunsch, nur nicht falsch zu urtheilen, eintritt, ein dem sicheren Urtheilen nachtheiliges Reflectiren (,zu langes Besinnen"). Jedoch geht aus den vorliegenden Bestimmungen sehon hervor, dass wenige Versuchspersonen mit vielen Urtheilen mehr richtige 
Fälle liefern, als viele mit wenigen Urtheilen für jede; denn 5 Personen mit 945 Proben lieferten 311 richtige Fälle, 12 Personen mit 1935 Proben 301 richtige Fälle. In ersterer Gruppe kamen im Durchschnitt 189,0 Urtheile, davon 62,2 genau richtige, auf jeden, in letzterer im Durchschnitt 94,6 Urtheile, davon nur 25,0 richtige auf jeden. Also gaben Wenige nach vielen Proben $32,9 \%$, Viele nach wenigen Proben für jeden nur 26,5\% richtige Fälle. Der Unterschied ist erwähnenswerth, weil bei einer so grossen Zahl von Einzelversuchen (2080) die Annahme eines Zufalls unwahrscheinlich und die eines guinstigen Einflusses der Uebung wahrscheinlich wird.

Aber der Einfluss individueller Verschiedenheiten ist nicht ansgeschlossen. Im Gegentheil individuelle Structurungleichheiten des Kopfes, wie ungleiche Dicke der Haut (mit den Haaren), des Schädels, ungleiche physische Beschaffenheit des Gehirns (als Schallleiters), müssen ebenso von Einfluss sein, wie ungleiches Urtheilsvermögen, also eine rein psychische Verschiedenheit der 17 Versuchsindividuen.

Das beste lieferte denn auch $36,8 \%$, das am wenigsten richtig urtheilende nur $18,7 \%$ richtige Fälle, jedoch 8 von den 17 iiber $30 \%$.

Um diesen Einfluss der Individualität, der beim Augenmaass bekanntlich ebenfalls hervortritt, festzustellen, bedarf es ebenso wie zum Beweise, dass Uebung auch hier die Anzahl der richtigen Fälle steigert, einer erheblich grösseren Anzahl von Versuchen, als bis jetzt vorliegen. --

Allen denjenigen, welche sich zu den vielen Bestimmungen mit der Schallhaube bereit fanden, sprechen wir schliesslich unseren verbindlichsten Dank aus.

\section{Erläuterung der Tafel IX.}

F'ig. 1. Transversalebene mit den vier Axen, 1) oben-unten (0- $u)$ von der Scheitelmitte zum Damm, verticale Axe, 2) rechts-links $(r-l)$ vom rechten Trommelfell zum linken, die transversale, frontale oder Quer-Axe, auch akustische oder Gehör- oder Hör-Axe genannt, 3) obenrechts-untenlinks $(o r-u l)$, vom rechten Scheitel nach der linken Schulter, 4) obenlinks-untenrechts (ol-ur) vom linken Scheitel nach der rechten Schulter. Winkel $=45^{\circ}$. 
Fig. 2. Sagittalebene oder Medianebene mit den vier Axen, 1) oben-unten $(0-u)$ wie in Fig. I, 1), vorn-hinten $(v-h)$ vom Nasenrücken (Mitte) zur Hinterkopfmitte, die sagittale Axe, 3) hintenoben-vornunten (ho-vu) vom Hinterscheitel oder Haarwirbel zum Kinn, 4) vornobenhintenunten $(v o-h u)$ von der Stirnmitte zur Nackenmitte. Winkel $=45^{0}$.

Fig. 3. Horizontalebene mit den vier Axen, 1) vorn-hinten $(v-h)$ wie in Fig. II, 2), links-rechts $(l-r)$ wie in Fig. I, 2), vornlinks-hintenrechts (vl-vr) von der linken Wange nach dem rechten Hinterkopf, 4) vornrechts-hintenlinks $(v r \cdot h l)$ von der rechten Wange nach dem linken Hinterkopf. Winkel $45^{\circ}$.

Fig. 4. Horizontalebene des um $45^{0}$ nach vorn, allein um die akustische Axe (l-r) gedrehten Kopfes mit den vier Axen: 1) vornoben-hintenunten $(v o-h u)$ von der Stirnmitte nach der Nackenmitte, 2) linksrechts (l-r) wie in Fig. I, 2), vornobenlinks-hintenuntenrechts (vol-hur) von der linken Stirn zum rechten Nacken, 4) vornobenrechts-hintenuntenlinks $(v o r-h u l)$ von der rechten Stirn zum linken Nacken. Winkel $54,7^{\circ}$ und $35,3^{\circ}$.

Fig. 5. Transversalebene des um $45^{\circ}$ nach vorn, allein um die akustische Axe (l-r) gedrehten Kopfes mit den vier Axen: 1) hintenoben-vornunten $(h o-v u)$ vom Haarwirbel oder der Hinterscheitelmitte zur Kinnmitte, 2) links-rechts (l-r) wie in Fig. I, 2), hintenobenrechts-vornuntenlinks (hor-vul) vom rechten Hinterscheitel (rechts vom Haarwirbel) zum linken Kinn, 4) hintenobenlinks-vornuntenrechts (hol-vur) vom linken Hinterscheitel (links vom Haarwirbel) zum rechten Kinn. Winkel $54,7^{\circ}$ und $35,3^{\circ}$.

In jeden der fünf Köpfe sind die Bogengänge, der Deutlichkeit halber etwas vergrössert, hineingezeichnet, so dass die im Text S. 611 besprochene ungleiche Erregung der Ampullennerven bei ungleicher Schallrichtung, während der Schall den Kopf durchsetzt, einigermaassen anschaulich gemacht ist, sofern in den Zeichnungen die 26 Schallrichtungen mit den Ebenen der Bogengänge ungleiche Winkel bilden und bald der eine, bald der andere zuerst und zumeist getroffen wird. Um sich genauer diese Verhältnisse vorzustellen, ist es empfehlenswerth, einen macerirten Schädel mit eröffnetem Felsenbein und ganz freigelegten knöchernen Bogengängen zu fixiren und die Axen an diese oder auch an die mit Metall ausgegossenen Bogengänge in natïrlicher Lage anzulegen, was ich nicht schwierig fand.

\section{Verzeichniss der Holzschnitte im Texte.}

Fig. 1. Die "Schallhaube“ (S. 589).

"2. Der enteckte entkantete Würfel (S. 591).

n. Das enteckte entkantete Oktaëder (S. 591).

\#. Das Axenmodell (S. 591).

” 5. Der „Schallglobus" (S. 592). 
Antive fic gas. Physiogie Ft. KL

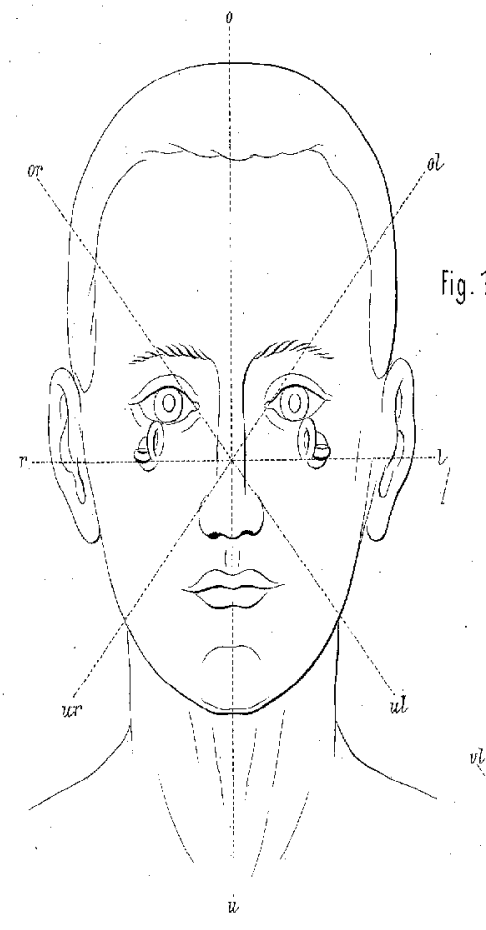

ig. 1.
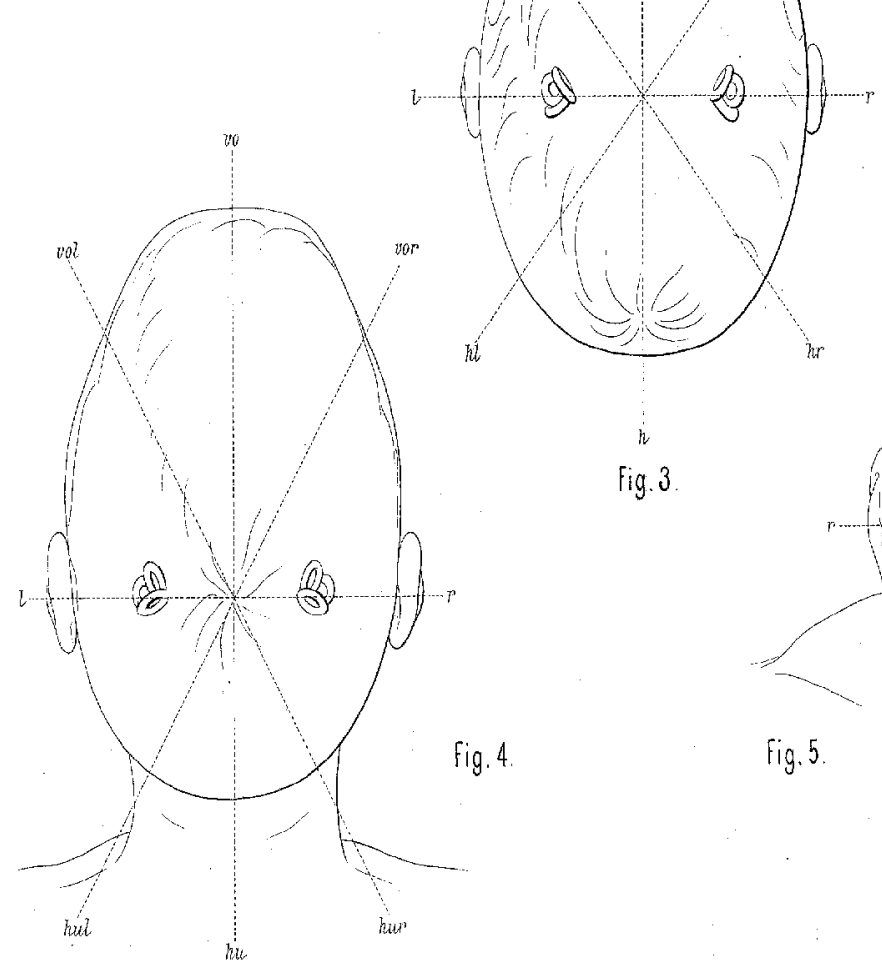

Fig. 4

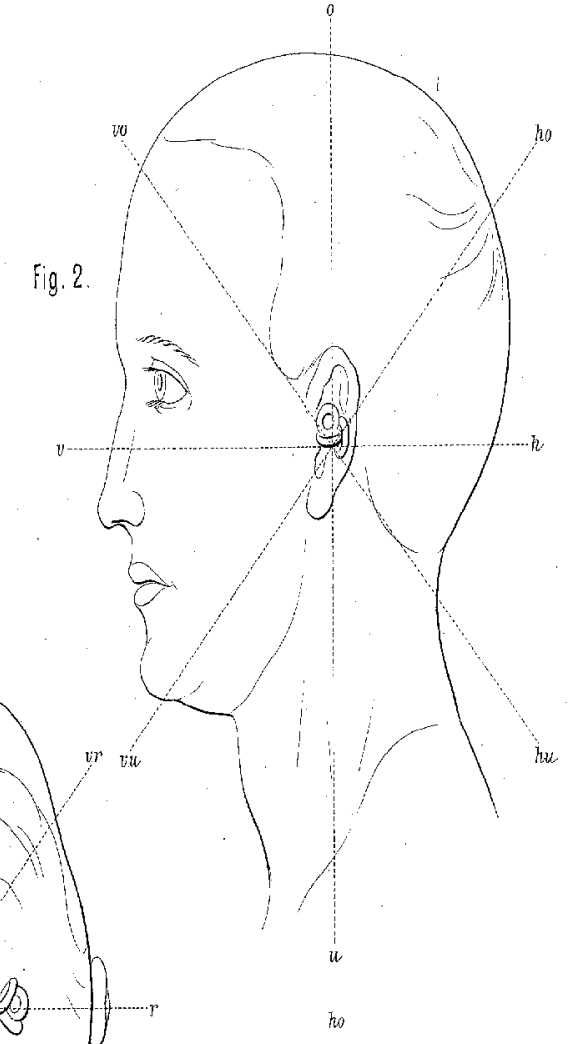

